\title{
An Object Model of String Field Theory and Derivation of Veneziano Amplitude
}

\author{
Holger B. Nielsen* \\ Niels Bohr Institute, University of Copenhagen, \\ 17 Belgdamsvej, DK 2100 Denmark \\ E-mail: hbech@nbi.dk \\ Masao Ninomiya \\ Advanced Mathematical Institute, Osaka City University, 3-3-138 Sugimoto, Sumiyashi-ku \\ 558-8585, Japan \\ E-mail: msninomiya@gmail.com
}

\begin{abstract}
We have proposed a new type of string field theory. The main point of the present article is to cure some technical troubles: missing two out three terms in Veneziano amplitude. Our novel string field theory, describes a theory with many strings in terms of "objects", which are not exactly, but close to Charles Thorn's string bits. The new point is that the objects in terms of which the universe states are constructed, and which have an essentially 26-momentum variable called $J^{\mu}$, can have essentially the energy $J^{0}$ be also negative. We get a long way in deriving in this model the Veneziano model and obtain all the three terms needed for a four point amplitude. This result strongly indicates that our novel string field theory is indeed string theory.
\end{abstract}

Corfu Summer Institute 2016 "School and Workshops on Elementary Particle Physics and Gravity" 31 August - 23 September, 2016

Corfu, Greece

${ }^{*}$ Speaker. 


\section{Introduction}

In order to describe a situation with several strings[1], [2], [3], [4], [5], [6], [7] you need a priori a string field theory[8] - second quantization - of the of the strings like in Kaku and Kikkawa[9], [10], [11], [12] or Witten theory[13], [14], [15], [16], [17]. But we have ourselves rather a description[18], [19], [20] (more similar to the string bit description of Charles Thorn[21]) in which the state of an arbitrary number of strings is described by relating it to a state of a very large number of what we call "objects", and which have degrees of freedom like free particles.

The basic steps in writing in our formalism the second quantized state/Hilbert vector for a given set of strings are the following:

1. To every string construct the "cyclic chain(s)"- one for an open string and two for a closed one- in principle for each "classical state" by wiriting the developing (single string) state in terms of the splitting $X^{\mu}(\sigma, \tau)=X_{R}^{\mu}(\tau-\sigma)+X_{L}^{\mu}(\tau+\sigma)$. Then the curves presented by $\dot{X}_{R}^{\mu}(\tau-\sigma)$ parametrized by $\tau-\sigma$ and by $\dot{X}_{L}^{\mu}(\tau+\sigma)$ parametrized by $\tau+\sigma$ in $25+1$ dimensional Minkowski space-time. For the open string there is a trick that actually these two curves naturally continue each other into just one closed curve/just one closed cyclic chain.

2. Next these "cyclic chains" (from 1.) are discretized into small bits which we call objects. Notice that it is in the "light corn" variables $\tau_{R}=\tau-\sigma$ and $\tau_{L}=\tau+\sigma \underline{\text { we make discretization }}$ into a lattice of "objects" (Not like Thorn in $\sigma$ ). Quantum mechanically some sophisticated trick of using only the even numbered lattice points=objects (to avoid noncommutation of the object variables).

3. Next the many string state is represented by a Hilbert vector in an a priori free massless scalar particle quantum field theory by acting on a certain vacuum state $|0\rangle$ with a creation operator for each even (numbered) "object" in any one of the set of strings the state of which is to be constructed.

So the reader should see that we have made a correspondence which to any state of an arbitrary number of strings let correspond a Hilbert vector in a massless free scalar quantum field theory. I.e. we manage to make our novel string field theory become an ordinary quantum field theory!

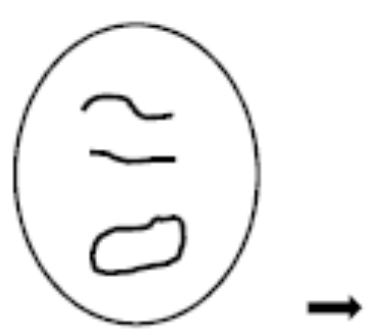

(a)

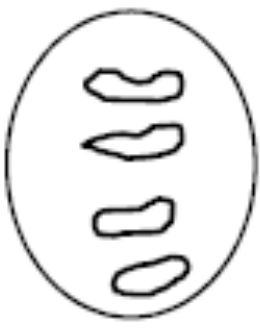

(b)

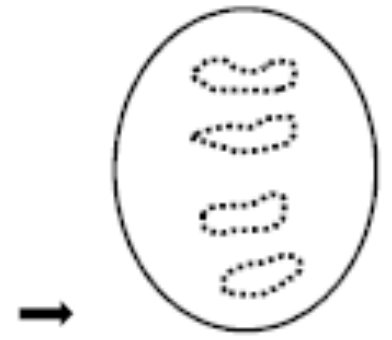

(c) 
Figure 1: Open and closed strings (a) to our object formalism (b) and (c)

From the previous worksof ours it is important to remember: We develop a formalism for description of an arbitrary number of strings (String Field Theory) by means of a Hilbert space formally with "objects" that are more like particles (they are "bits" in a technically a little different way from Thorn's).

In terms of our "objects" (bits) the second quantized string world get totally static, scattering becomes a fake: the scattering amplitude becomes just the overlap of the initial with final states!

We get, after some technical trouble, the S-matrix = the overlap between initial and final string states to be the Veneziano amplitude.

It is very crucial that the objects must be able to have energy of both signs.

So one piece of a cyclic chain can cancel another piece completely!

And thus pairs of compensating pieces of chains of objects may be phantazised where-ever it may be.

"String" comes ONLY in via the initial (and final state) conditions.

\subsection{Our SFT model Equivalent to String Theory}

The final SFT-model(string field theory model)[13] of ours is described by the Fock space for massless non-interacting scalar bosons in $25+1$ dimensions. That is to say it is described by a Hilbert space, which is generated by a series of creation $a^{+}(\vec{p})$ and destruction $a(\vec{p})$ operators for scalar particels with 25 -momenta $\vec{p}$, which can act successively on a zero-particle state $|0\rangle$. That is to sey that the typical states in the Fock space - or the Hilbert space describing the world state are

$$
a^{+}\left(\vec{p}_{1}\right) a^{+}\left(\vec{p}_{2}\right) \cdots a^{+}\left(\vec{p}_{n}\right)|0\rangle
$$


In the language, which we use, we call the scalar particles "even objects" and denote their momenta by $J^{\mu}$ instead of $p^{\mu}$ (well really we only consider the conjugate momenta for the transverse components $i=1,2, \ldots, 24$, i.e. $\left.J^{\mu}\right)$.

\subsection{Relation To Charles Thorn's String Bits}

We deviate from the original Charles Thorn's idea[12] of describing the string as a chain of string-bits by Thorn using bits corresponding to small pieces of the string sigma-variable, while we take the step into bits- which we then to distinguish can call "objects" - instead after, we have split the solution into right and left mover and thus rather put into bits or now to distinguish objects the right-mover variable " $\tau-\sigma$ " or the left mover one " $\tau+\sigma$ ".

Actually Thorn has begun to do the same as we later at least for fermion modes.
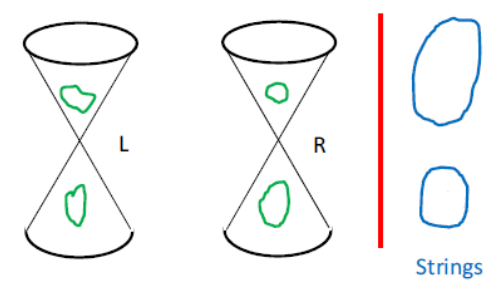

(a) For theory with only closed strings

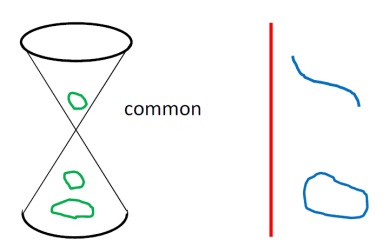

(b) For theories with also open strings

Figure 2: Chains of objects on the light cone in the $25+1$ dimensional Minkowski space. (a) is depicted on the closed strings in terms of the left and right movers(b) The left and right movers of the open and closed strings are written together. 


\subsection{Translation from strings to "Cyclic Chains"}

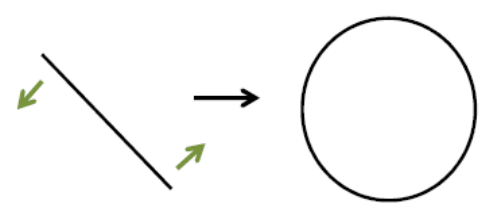

(a) Open string corresponds to topologically circular figure: The cyclic chain

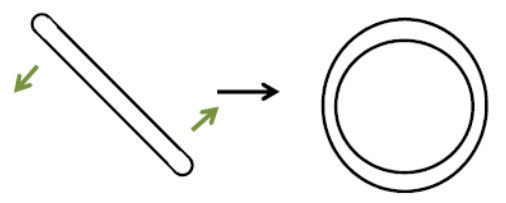

(b) Closed string corresponds to 2 different cyclic chain

Figure 3: Transition from strings to "cyclic chains"

As a very simple example of a classically described open strings, we can think of a string rotating as a stiff stick around its middle point. Say for simplicity that it is at rest and that we choose a gauge/a parametrization so that the energy is used to determine the parameter $\sigma$ along the string, so that an infinitesimal interval do in $\sigma$ just has the energy

$$
d E=d \sigma
$$

If $r$ denotes the distance from the middle point $C$ to the point with coordinate $\sigma$, and $R$ denotes half the geometrical length (in target space) of the (open) string, and $\omega$ the rotation rate, then the energy density at the point $\sigma$ is

$$
\frac{d \sigma}{d r}=\frac{d E}{d r}=\frac{1}{2 \pi \alpha^{\prime} \sqrt{1-v^{2}}}=\frac{1}{2 \pi \alpha^{\prime} \sqrt{1-\omega^{2} r^{2}}}
$$

Remembering

$$
\frac{d}{d(\omega r)} \arcsin (\omega r)=\frac{1}{\sqrt{1-(\omega r)^{2}}}
$$

thus

$$
\begin{aligned}
d \sigma & =\frac{d r}{2 \pi \alpha^{\prime}} \frac{d}{d(r \omega)} \arcsin (\omega r) \\
& =\frac{1}{2 \pi \alpha^{\prime} \omega} d \operatorname{arcsine}(\omega r)
\end{aligned}
$$

so that by integrating we may take the integration constant to get

$$
\sigma=\frac{1}{2 \pi \alpha^{\prime} \omega} \operatorname{arcsine}(\omega r)
$$


or

$$
r=\frac{1}{\omega} \sin \left(\omega 2 \pi \alpha^{\prime} \sigma\right)
$$

The half length $R$ of the (open) string is of course the maximal achievable

$$
R=\frac{1}{\omega} .
$$

Let us identify the usual internal string time $\tau$ with the target space time essentially and let us take the plane in which the string rotates to be the $X^{1} X^{2}$-plane. Then while the coordinates $X^{3}=X^{4}=$ $\ldots=X^{25}=0$ of course we have

$$
\begin{aligned}
X^{1}(\sigma, \tau) & =\cos \left(\omega 2 \pi \alpha^{\prime} \tau\right) r \\
& =\frac{1}{\omega} \cos \left(\omega 2 \pi \alpha^{\prime} \tau\right) \sin \left(2 \pi \alpha^{\prime} \omega \sigma\right) \\
X^{2}(\sigma, \tau) & =\frac{\sin \left(\omega 2 \pi \alpha^{\prime} \tau\right) \sin \left(2 \pi \alpha^{\prime} \sigma \omega\right)}{\omega}
\end{aligned}
$$

To have a hope of having made a proper conformal gauge choice we take $\tau=\frac{t}{2 \pi \alpha^{\prime}}$.

If the solution ansatz which we have here made on physical ground is indeed a solution to the internal D'Alembertian equation

$$
\left(\frac{\partial^{2}}{\partial \tau^{2}}-\frac{\partial}{\partial \sigma^{2}}\right) X^{\mu}(\sigma, \tau)=0
$$

it shall be possible to resolve the $X^{\mu}$ into right and left mover like

$$
X^{\mu}(\sigma, \tau)=X_{R}^{\mu}(\tau-\sigma)+X_{L}^{\mu}(\tau+\sigma)
$$

Indeed it is easy to use the formulas for taking $\sin$ and $\cos$ for sums of variables such as

$$
\sin \left(\omega \cdot 2 \pi \alpha^{\prime}(\tau \pm \sigma)\right)=\sin \left(2 \omega \pi \alpha^{\prime} \tau\right) \cos \left(2 \omega \pi \alpha^{\prime} \sigma\right) \pm \cos \left(2 \omega \pi \alpha^{\prime} \tau\right) \sin \left(2 \omega \pi \alpha^{\prime} \sigma\right)
$$

and

$$
\cos \left(\omega 2 \pi \alpha^{\prime}(\tau+\sigma)\right)=\cos \left(2 \omega \pi \alpha^{\prime} \tau\right) \cos \left(2 \omega \pi \alpha^{\prime} \sigma\right) \mp \sin \left(\omega 2 \alpha^{\prime} \pi \tau\right) \sin \left(\omega 2 \alpha^{\prime} \pi \sigma\right)
$$

to rewrite e.g.

$$
\begin{aligned}
X^{1}(\sigma, \tau) & =\frac{1}{\omega} \cos \left(\omega 2 \pi \alpha^{\prime} \tau\right) \sin \left(\omega 2 \pi \alpha^{\prime} \sigma\right) \\
& =\frac{1}{2 \omega}\left(\sin \left(\omega 2 \pi \alpha^{\prime}(\tau+\sigma)\right)-\sin \left(\omega 2 \pi \alpha^{\prime}(\tau-\sigma)\right)\right. \\
& =X_{L}^{1}(\tau+\sigma)+X_{R}^{1}(\tau-\sigma)
\end{aligned}
$$

where then

$$
X_{R}^{1}(\tau-\sigma)=\frac{-1}{2 \omega} \sin \left(\omega 2 \pi \alpha^{\prime}(\tau-\sigma)\right)
$$

and

$$
X_{L}^{1}(\tau+\sigma)=\frac{1}{2 \omega} \sin \left(\omega 2 \pi \alpha^{\prime}(\tau+\sigma)\right)
$$


Similarly we can write

$$
\begin{aligned}
X^{2}(\sigma, \tau) & =\frac{\sin \left(\omega 2 \pi \alpha^{\prime} \tau\right) \sin \left(\omega 2 \pi \alpha^{\prime} \sigma\right)}{\omega} \\
& =\frac{1}{2 \omega}\left(\sin \left(\omega 2 \pi \alpha^{\prime}(\tau+\sigma)\right)+\sin \left(\omega 2 \pi \alpha^{\prime}(\tau-\sigma)\right)\right. \\
& =X_{L}^{2}(\tau+\sigma)+X_{R}^{2}(\tau-\sigma)
\end{aligned}
$$

where

$$
\begin{aligned}
& X_{L}^{2}(\tau-\sigma)=\frac{1}{2 \omega} \sin \left(\omega 2 \pi \alpha^{\prime}(\tau-\sigma)\right) \\
& X_{R}^{2}(\tau+\sigma)=\frac{1}{2 \omega} \sin \left(\omega 2 \pi \alpha^{\prime}(\tau+\sigma)\right)
\end{aligned}
$$

At the ends of the string corresponding in the above notation to $\omega 2 \pi \alpha^{\prime} \sigma= \pm \frac{\pi}{2}$ we shall have that the tension in the string

$$
\text { "tension" } \propto X^{\prime \mu}(\sigma, \tau)=-\dot{X}_{R}(\tau-\sigma)+\dot{X}_{L}(\tau+\sigma)
$$

shall be zero, since there is nothing further out, in which it can pull.

Calling

$$
\begin{aligned}
& \tau_{R}=\tau+\sigma \\
& \tau_{L}=\tau-\sigma
\end{aligned}
$$

We could write our above formulas

$$
\begin{aligned}
& X_{R}^{1}\left(\tau_{R}\right)=-\frac{1}{2 \omega} \sin \left(\omega 2 \pi \alpha^{\prime} \tau_{R}\right) \\
& X_{L}^{1}\left(\tau_{L}\right)=\frac{1}{2 \omega} \sin \left(\omega 2 \pi \alpha^{\prime} \tau_{L}\right) \\
& X_{R}^{2}\left(\tau_{R}\right)=\frac{1}{2 \omega} \sin \left(\omega 2 \pi \alpha^{\prime} \tau_{R}\right) \\
& X_{L}^{2}\left(\tau_{L}\right)=\frac{1}{2 \omega} \sin \left(\omega 2 \pi \alpha^{\prime} \tau_{L}\right)
\end{aligned}
$$

and boundary conditions of no tension becomes

$$
\dot{X}_{L}^{\mu}\left(\tau_{L}=\tau+\frac{\pi}{2 \cdot 2 \pi \alpha^{\prime} \omega}\right)=\dot{X}_{R}^{\mu}\left(\tau_{R}=\tau-\frac{\pi}{2 \cdot 2 \pi \alpha^{\prime} \omega}\right)
$$

the end of the string having $\sigma=-\frac{\pi}{2}$ obtain

$$
\dot{X}_{L}^{\mu}\left(\tau_{L}=\tau-\frac{\pi}{2 \omega 2 \pi \alpha^{\prime}}\right)=\dot{X}_{R}^{\mu}\left(\tau_{R}=\tau+\frac{\pi}{2 \omega 2 \pi \alpha^{\prime}}\right)
$$

For $\mu=1$ for example we see that these conditions are true because a shift in the $\tau$-argument $\tau_{R}$ or $\tau_{L}$ by 2 times $\frac{\pi}{2 \cdot \omega 2 \pi \alpha^{\prime}}$ corresponds to a shift by $\pi$ in the argument of the sine and that gives just the sine shift needed because $X_{R}^{1}\left(\tau_{R}\right)=-\frac{1}{2 \omega} \sin \left(\omega 2 \pi \alpha^{\prime} \tau_{R}\right)$ while $X_{L}^{1}\left(\tau_{L}\right)=\frac{1}{2 \omega} \sin \left(\omega 2 \pi \alpha^{\prime} \tau_{L}\right)$. 
Apart from some shift in the argument the $X_{R}^{\mu}$ and $X_{R}^{\mu}$ are basically the same functional form - as we also see in our example - due to the boundary condition(s) at the end of the string. Because we have at present a bit bad notation used $\sigma=\frac{\pi}{2 \cdot 2 \pi \alpha^{\prime} \omega}$ at one of the ends we get in fact

$$
\dot{X}_{L}^{\mu}\left(\tau_{L}\right)=\dot{X}_{R}\left(\tau_{R}=\tau_{L}+\frac{\pi}{2 \pi \alpha^{\prime} \omega}\right)
$$

If we had the end to have $\sigma=0$ we would have simply gotten

$$
\dot{X}_{L}^{\mu}\left(\tau_{L}\right)=\dot{X}_{R}\left(\tau_{R}=\tau_{L}\right)(\text { for } \sigma=0 \text { at end). }
$$

In any case of course we only need to use either $\dot{X}_{R}^{\mu}$ or $\dot{X}_{L}^{\mu}$ since they are of the same form.

\subsection{The closed string}

On the second translation on the figure we have taken as example a closed string in the configuration wherein it is put back and forth along the same piece of line, and it - really meaning its two pieces - rotate just like the open string just considered. Now the seeming end points are just accidental but not truly physically. In this case of closed we shall not identify the right and left movers. Rather each of them give rise to its own "cyclic chain". Therefore we have for this drawn two independent (although they happen to have the same coordinates) "cyclic chains". Let us stress the rule: To an open string corresponds only one cyclic chain, while to a closed string there corresponds two, one for the right mover modes and one for the left.

Concerning the above discussion it should be noted that we considered

$$
\begin{aligned}
& \dot{X}_{R}^{\mu}\left(\tau_{R}\right)=\frac{d X_{R}^{\mu}}{d \tau_{R}} \\
& \dot{X}_{L}\left(\tau_{L}\right)=\frac{d X_{L}^{\mu}}{d \tau_{L}}
\end{aligned}
$$

rather than $X_{R}^{\mu}\left(\tau_{R}\right)$ and $X_{L}^{\mu}\left(\tau_{L}\right)$ themselves - and that is something we for technical reasons, have decided to do in our Novel SFT - Had we not done that, the arguing away having both $X_{L}^{\mu}$ and $X_{R}^{\mu}$ in the open string would not have worked. So it were a quite important point to make this differentiation!

For illustration of our formulation/model for our novel string field theory you shall imagine drawing in 25 or 26 dimensional perspective the right mover field $X_{R}$ differentiated with respect to its variable $\tau-\sigma$, thinking classically.

To each open string right mover derivative is a 26 -vector being a periodic function with the period used for $\sigma$. The boundary condition at the end ensures that right and left mover derivatives are equal for the open string.

Thus we get to the open string a corresponding topologically circular figure the "cyclic chain". For a closed string one can both imagine drawing the right and the left mover and they become two in general different closed curve images (= two "cyclic chains").

For a single string in the "conformal gauge" we have the well-known

$$
\left(\partial_{\tau}^{2}-\partial_{\sigma}^{2}\right) X^{\mu}(\sigma, \tau)=0
$$


and solve it by the splitting

$$
X^{\mu}(\sigma, \tau)=X_{R}^{\mu}(\tau-\sigma)+X_{L}^{\mu}(\tau+\sigma)=X_{R}^{\mu}\left(\tau_{R}\right)+X_{L}^{\mu}\left(\tau_{L}\right)
$$

As introduction to our Novel String Field Theory we shall imagine - and let us first think classically - that for each string development in time - in Minkowski space - draw a to such a moving/oscillating string corresponding image of the $\tau_{R}=\tau-\sigma$ respective $\tau_{L}=\tau+\sigma$ derivatives $\dot{X}_{R}^{\mu}\left(\tau_{R}\right)$ and $\dot{X}_{L}^{\mu}\left(\tau_{L}\right)$ of these $X_{R}^{\mu}\left(\tau_{R}\right)$ and $X_{L}^{\mu}\left(\tau_{L}\right)$.

Because of the periodicity for finite size strings the two images of $X_{R}$ and $X_{L}$ will be closed curves, called "cyclic chains".

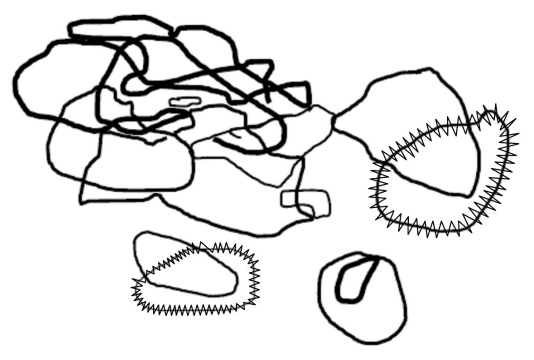

Figure 4: The images of $X_{R}$ and $X_{L}$ are represented by the closed curves called cyclic chains. The all curves do not have ends.

The meaning of the foregoing figure with the net of curves is, that there is in a perspectively drawn Minkowski space of $26=25+1$ dimensions (in bosonic string case) a point on the net each time some one of all strings present in Universe for some value of $\tau_{R}=\tau-\sigma$ or $\tau_{L}=\tau+\sigma$ the respective derivatives $\dot{X}_{R}^{\mu}\left(\tau_{R}\right)$ or $\dot{X}_{L}^{\mu}\left(\tau_{L}\right)$ of the string space time position field $X^{\mu}(\tau, \sigma)$ take their vectorial value equal to that point. This net of curves is thought classically at first: i.e. $\dot{X}_{R}^{\mu}\left(\tau_{R}\right)$ and $\dot{X}_{L}^{\mu}\left(\tau_{L}\right)$ have meaningful vectorial values once the last bit of gauge choice has been chosen.

\subsection{One Open string would contribute say the slim closed curve contained in the net}

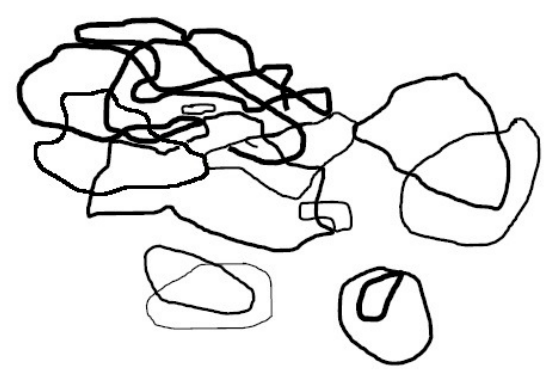

Figure 5: One open string depicted by the slim closed curve contributes in the net. 
For an open string one has at both end-points (say $\sigma=0,2 \pi$ )

$$
X^{\prime \mu}=-\dot{X}_{R}^{\mu}+\dot{X}_{L}^{\mu}=0
$$

Since this must be true for all $\tau$, it is enough information to deduce that for the open string

$$
\dot{X}_{R}^{\mu}(\tau)=\dot{X}_{L}^{\mu}(\tau)
$$

Thus at any moment of $\tau$ there is for each open string a closed circle, a "cyclic chain", of $\dot{X}_{R}^{\mu}$ or $\dot{X}_{L}^{\mu}$, so that we get for each open string a closed circle (the cyclic chain) of image points in the (perspectively imagined) $26=25+1$ dimensional space on the figure.

\subsection{We Add one more - now wavy curve - Open string Contribution, a Cyclic Chain}

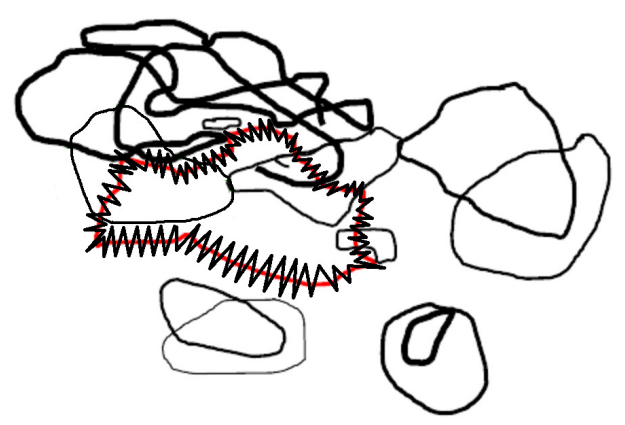

Figure 6: One more open string contribution is added as a wavy closed curve in the cyclic chain. All curves have no ends.

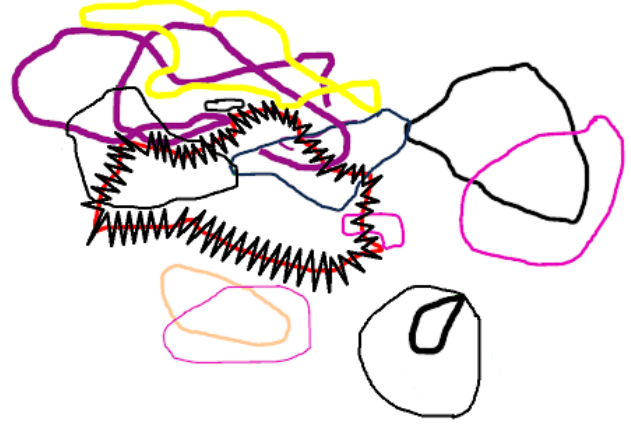

Figure 7: The whole set of all the right or left mover derivatives are symbolized by the thin-curved network. They can be interpreted as unification of a series of closed cycles that close each other.

\subsection{Comments on Our net of Cyclic Chains Representing String Via their Right and Left mover Derivatives}

The whole set of all the right or left mover derivatives symbolized by the thin-curved network, can be interpreted as coming from various cyclic chains associated with various open string, or 
some pairs of them could correspond to closed strings.

We illustrated by various colors and thicknesses of the curves, how the image of all the right and left mover derivatives could be divided into contributions e.g. from different open strings. (some pairs of cyclic chains potentially correspond to just the same closed string instead of to two open ones).

We stress that the division into contributions from different cyclic chains, different open strings say, is not unique!

\subsection{Further Comments on Novel String Field Theory Model}

For seeing our model, our novel string field theory, it is the crucial point to imagine that the cyclic chains shown on our figures here in the $25+1=26$ dimensional space(-time) should be thought of as series of "objects" meaning a discretization of the cyclic chains into particle-like objects, that can be created or annihilated by creation and annihilation operators. So in this creation and annihilation we already now think quantum mechanically.

Thus the net of cyclic chains is truly thought of as represented by the effect of a lot of actions with creation or annihilation operators on a "background state"(=vacuum)

The spirit of our novel string field theory is most quickly presented by simply replacing the sites of $\dot{X}_{L}^{\mu}$ and $\dot{X}_{R}^{\mu}$ values representing a state of a universe with a number of open and closed strings called $I m$ by a representation by a Hilbert space vector in a Hilbert space, in which what we call "objects" can be created and annihilated. The main point is the replacement

$$
\left.I m \rightarrow \prod_{J_{\in}^{\mu} I m} a^{+}\left(J^{\mu}\right) \mid \text { "vac" }\right\rangle
$$

written very shortly.

Here $J^{\mu}$ denotes a Minkowski space point and the product runs over those $J^{\mu}$,s which lie in the union set Im.

There is, however, a series of technical troubles and the simple replacement

$$
I m \rightarrow \prod_{J_{\in} I m} a^{+}(J)|" v a c "\rangle
$$

is to be considered an oversimplified pedagogical presentation, being quick correct.

First of all formulating a product of creation operators $a^{+}\left(J^{\mu}\right)$ requires that the product runs over a discrete set rather than a continuous set as $I m$. So really we rather should say

$$
\left.I m \rightarrow \prod_{I_{\in} \text { discretized } I m} a^{+}\left(J^{\mu}(I)\right) \mid \text { "vac" }\right\rangle
$$

where we have split the "continuous" set Im into a large number of small pieces (or intervals in $\tau_{R}$ or $\tau_{L}$ 's) enumerated by essentially an integer $I$.

Secondly what we would achieve then would not be a true quantum theory because $\dot{X}_{R}^{\mu}\left(\tau_{R}\right)$ say in quantum single string theory does not commute with itself for different $\tau_{R^{-}}$alues

$$
\left[\dot{X}_{R}^{\mu}\left(\tau_{R}\right), \dot{X}_{R}^{v}\left(\tau_{R}^{\prime}\right)\right]=i \delta^{\prime}\left(\tau_{R}-\tau_{R}^{\prime}\right)
$$


To be able to properly quantize both the single object system and second quantize, the discretized $J^{\mu}(I) \stackrel{\propto}{\sim} \dot{X}_{R}^{\mu}\left(\tau_{R}\right)$ should be so that they mutually commute. The trick to achieve such commutation since long proposed for our novel string field theory was to only use to give creation operators $a^{+}\left(J^{\mu}(I)\right)$ for the even I's. That is to say that the true expression becomes

$$
\left.I m \rightarrow\left(\prod_{I_{\epsilon} \text { discretized Im and I even }} a^{+}\left(J^{\mu}(I)\right)\right) \mid \text { “vac" }\right\rangle
$$

Here the important point is had the $I$ variable only runs over every other of the at first twice as large number of bits/objects. Because the commutator $i \delta^{\prime}\left(T_{R}-T_{R}^{\prime}\right)$ after discretization gives lack of commutation between neighboring bits (or objects) we can achieve full commutation if we leave out every other of the bits, we call the left out ones the odd and denote them by odd integers.

A third minor technical problem is that we should like to have the objects be their own antiparticles (they should be "Majorana" so to speak) I.e.

$$
a^{+}\left(J^{\mu}\right)=a\left(-J^{\mu}\right) .
$$

But if so we would with a "normal" quantization get

$$
\begin{array}{r}
a^{+}\left(J^{\mu}\right)|" v a c "\rangle=0 \\
\text { for } J^{0}<0 .
\end{array}
$$

In fact it were the trouble of our calculation in which we only obtained one of the three terms in the Veneziano amplitude. To avoid that we should even be able add some negative energy to the vacuum $\mid$ "vac" $\rangle$ i.e. we want

$$
\begin{array}{r}
a^{+}\left(J^{\mu}\right)|“ v a c "\rangle \neq 0 \\
\left(\text { even for } J^{0}<0\right)
\end{array}
$$

even when what we interpret $J^{0}$ the energy is negative.

It is this problem that shall be solved by using as vacuum a state with the property that $a^{+}\left(J^{\mu}\right)|0\rangle$ shoud not be zero.We call such a vacuum "the rough Dirac sea".

\subsection{The Rough Dirac Sea}

At first one would be tempted to think of the vacuum or "background state" for the objects as a state in which objects with positive energy (if that makes any sense) were the only ones possible to produce, but...:

Even though energy of a single object can be given a meaning, we shall assume that the "back ground state" for the second quantized objects-theory is not of the simple type that can only be modified to make the sum of the energies of the objects larger! Rather you can also add to it negative energy.

This is analogous to what we call the "rough Dirac sea"(see Appendix)

On the last figure we show putting some arrows, how to construct the points or small pieces of the string time track -i.e. the surface in Minkowski space through which the string in question passes: To each pair of small bits on the cyclical chain corresponds a little area on the space- time 
track of the string.

The string time track is a two-dimensional manifold and thus one needs two one-dimensional parameters to parametrize it. We use for the open string the same cyclic chain as being both parameters (two different point on the cyclic chain), while we for the closed string we use two different cyclic chains.

\subsection{Illustration of Connection to the String}

We have put in on the picture fig. 8 of the curve a narrow arrow. Such an arrow corresponds to a point on the string time track, or rather one point for each period of the string motion, in the sense that a couple of tangent vectors spanning the tangent to the string time track at the point in question are given by the two points in $25+1=26$ space(-time) at the two ends of the (double)arrow.

To obtain all the time track points of an open string modulo periodicity you must take all the arrows that can connect two points on the cyclical chain describing the open string in question.

To obtain those for a closed string you must use all the arrows connecting one point on one of the two cyclic chains to the other one.

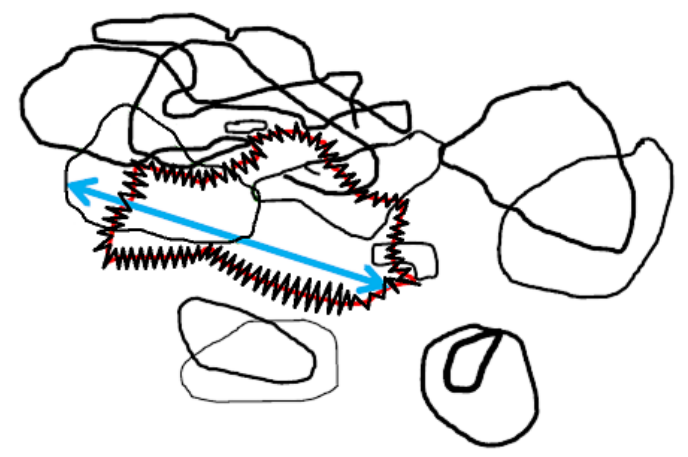

Figure 8: Imagining putting the double around to corneot all rears of one point on the blue and one on the red it would come through all the time track points on a closed string. For each rear run through the $\dot{X}_{R}^{\mu} \hat{a} \breve{A} Z$ s on the two rounds flax the tangent plane to the time track of the closed string.

On the figure illustrating the closed string in terms of two cyclical chains you obtain the tangent- basis vectors of the various infinitesimal pieces of the closed string time track by going through all possible arrows connecting one point on the cyclic chain, green, and one on the cyclic chain, red.

Again we get a two-dimensional time-track of a string -now closed- by having it parametrized by two parameters running along cyclic chains. But for the closed string these two parameters run on two different cyclic chains.

What happens if we first by creation or annihilation operator insert a piece of cyclic chain with one momentum distribution and then add the one with just the opposite one ? Actually they cancel and it becomes as if nothing had been done.

This opens up a strange possibility for inserting the cyclic chains corresponding to a couple of say open strings: We could let a piece of the cyclic chain corresponding to one of the two strings happen to be just the "opposite" of a piece of the cyclic chain for the other open string. 
In that case inserting the cyclic chains corresponding to the two open strings leads to there being two pieces of cyclic chains canceling each other....

And thus the final state in our $25+1=26$ dimensional space for "objects" would have got zero objects along the piece of cancellation. And the latter would not be marked in our Hilbert space for second quantized object..

One would only there "see" the pieces that were NOT canceled.

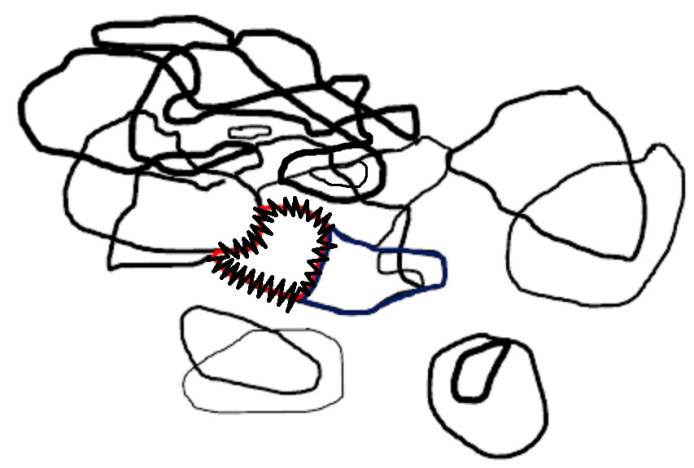

Figure 9: Illustration of how a piece of chain with two opposite curves of objects can go into the patern of cyclically orders chains. On this figure the piece with compensation as piece colored in two shades.

Having in mind the possibility of canceling two "opposite pieces of cyclic chain we can without any trace in our second quantized object-state have two (oppositely oriented but otherwise locally in the same state) pieces of the to the blue and the red cyclic chains present.

On the above figure these canceling pieces are illustrated with the curve where the blue and the red curves follow each other.

That there along this piece is no sign of the cyclic chains in the object-description is illustrated by there being no thin black curve along this piece before we drew in the red and the blue circles (cyclic chains) otherwhere on top of the thin black curves illustrating the second quantized object-state.

\section{The state of several strings}

At first -and that we did in our earlier work- in a Bled proceedings[18]- one would simply take the second quantized Hilbert space state as the incomming two particle state $\mid \vec{p}_{1}, \vec{p}_{2}$, inc $\rangle$ to be the one obtained from the object formulation vacuum $|0\rangle$ by first acting with the creation operator for the cyclically ordered chain.

$$
\begin{gathered}
C^{+}(\text {string } 1)=\int_{\text {around }} \Psi\left(J^{\mu}(0), J^{\mu}(2), J^{\mu}(N-2)\right) \\
\text { cyclic } \\
\text { chain } \\
I_{e} \text { even } \\
\cdot a^{+}\left(J^{\mu}(0)\right) a^{+}\left(J^{\mu}(2)\right) \cdots a^{+}\left(J^{\mu}(N-2)\right) \prod_{I_{e}^{\text {even }}} d J\left(I_{e}\right)
\end{gathered}
$$


on this vacuum $|0\rangle$ and then successively act with the analogous creation operator for string 2 , say $C$ (string 2). I.e. the description in our picture should be

$$
C^{+} \text {(string2) } C^{+}(\text {string } 1)|0\rangle
$$

\subsection{A formalism of Replacement of ghost}

If we imagine working with formulation with ghosts the $J^{\mu}\left(I_{e}\right)$ 's must be replaced by constructions such as $\left(J^{\mu}\left(I_{e}\right), \uparrow, \downarrow\right)$ or $\left(J^{\mu}\left(I_{e}\right), \downarrow, \downarrow\right)$ also involving ghost (but that is not so important just now).

If one works with our old infinite momentum frame it would only be the transverse components and you would write instead

$$
C^{+}(\text {string1 })=\int \Psi\left(\vec{J}_{T}(0), \vec{J}_{T}(2), \ldots, \vec{J}_{T}(N-2)\right) \prod_{I_{e}}\left(a^{+} \vec{J}\left(I_{e}\right)\right)\left(d \vec{J}_{T}\left(I_{e}\right)\right)
$$

If our creation operators for the objects like $a^{+}\left(\vec{J}_{T}\left(I_{e}\right)\right)$ all add say a positive energy rather than like the ones in say the BRST formalism $a^{+}\left(J^{\mu}\left(I_{e}\right)\right.$, ghost $)$, then there is no way that the products of a couple of creation operators for objects could be simplified. If we however have operators like $a^{+}\left(J^{\mu}\left(I_{e}\right), \uparrow, \downarrow\right)$ that are a priori able to bring energy and momentum of any sign, e.g. also negative energy $J^{0}$, then there is opened the possibility that the action of a couple of them

$$
a^{+}\left(J^{\mu}\left(I_{e}\right), \cdot, \cdot\right) a^{+}\left(-J^{\mu}\left(I_{e}\right), \cdot, \cdot\right) \propto 1
$$

could act proportional to a c-number!

This is the crucial progress by not fixing energy nor longitudinal momenta to be positive. For this to work it is crucial that two opposite 26-J creation operators both are nontrivial.

i.e. both say

$$
a^{+}\left(J^{\mu}, \cdot, \cdot\right)|0\rangle \neq 0
$$

and

$$
a^{+}\left(-J^{\mu}, \cdot, \cdot\right)|0\rangle \neq 0
$$

That requires a "rough Dirac sea". But provided - as we think we have - we have provided such nontrivial but opposite operator a "Majorana" boson theory for the objects say then one of the two operators $a^{+}\left(J^{\mu}, \cdot, \cdot\right)$ and $a^{+}\left(-J^{\mu}, \cdot, \cdot\right)$ may be considered the annihilation operator for the particle / here object created by the other one. Thus indeed we may argue

$$
a^{+}\left(-J^{\mu}, \cdot, \cdot\right) a^{+}\left(J^{\mu}, \cdot, \cdot\right)|0\rangle=|0\rangle
$$

so that the product acts as the unit operator. With continuum normalization one may have $\delta$ functions, but let us postpone this issue.

This means that in our "rough Dirac sea" picture we have to count that "oppoiste" (meaning opposite $J^{\mu}$, i.e. $J^{\mu}$ and $-J^{\mu}$ creation operators multiplied with each other can be replace -by calculation - by just unit operator. 


\subsection{A trick of calculating the wave function}

Now we should remember that our crucial trick to calculate the wave functions $\Psi_{1}$, and $\Psi_{2}$ for out strings 1 and 2 was to express them by means of imaginary $\tau$ functional integrals -so as to let only the ground state of the strings survive -.

If a couple of strings have it so that their associated creation operator cyclic chains can partly annihilate in the sense of giving unit operators as just described, then rather than being left in the two-string-describing state these objects can be removed provided they are "opposite".

The calculation of the amplitude for what the removing object creation operators can be well now by a functional integral for the complex (=imaginary $\tau$ ) developments (of the cyclically ordered chains) but with the extra rule: Piece(s) of the edge for string 1 half cylinder could be glued together with piece(s) of the edge for the half cylinder for string 2 :

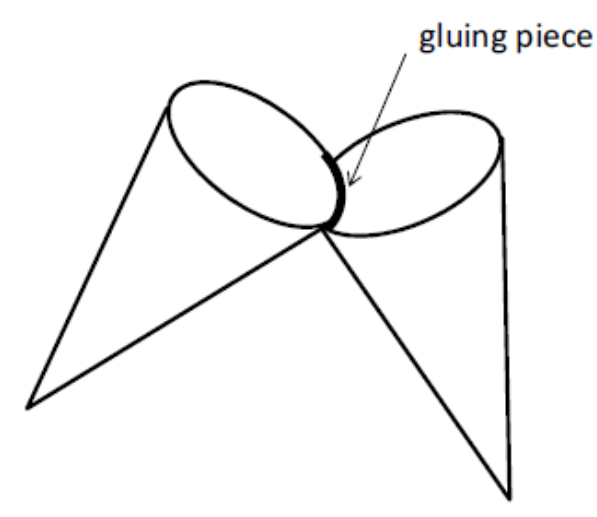

Figure 10: The two cones can be glued together at the common edges.

We would then -being closer to the classical solution - rather think of the picture

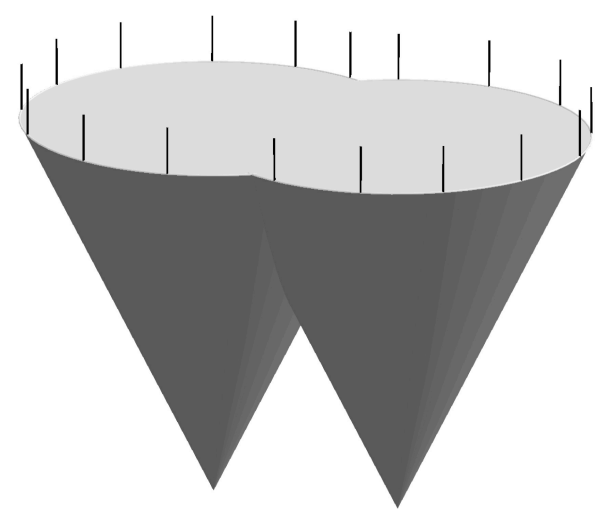

Figure 11: The two cones can have a common edge as depicted. 
as describing the functional integral to derive the resulting true object description for two string state

$$
\mid \text { str.1,str.2, } \underset{\text { com }}{\text { in }}\rangle=C^{+}(\text {string } 2) C^{+}(\text {string } 1)|0\rangle
$$

The possibility that there were nothing annihilated should not be neglected, since it is not necessarily negligible. So rather symbolically we can write these two terms as on figure 12 .

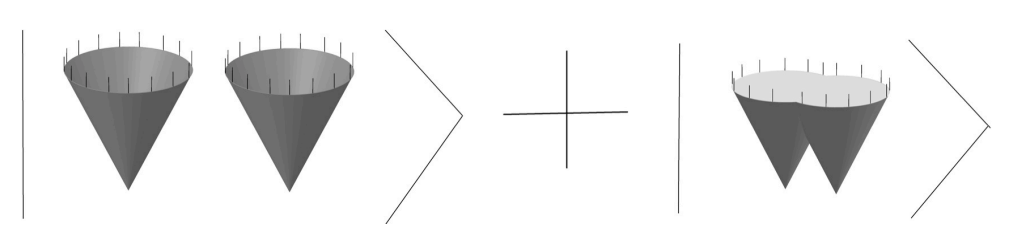

Figure 12: Two string states are sum of two state vectors as expressed in the figure.

These more complicated terms are obtained by taking from each of two incoming strings; string 1 and string 2 more than one piece of their cyclic chains and then we use the rule

$$
a^{+}\left(J^{\mu}, \cdot, \cdot\right) a^{+}\left(-J^{\mu}, \cdot, \cdot\right) \sim 1
$$

along these -more than one- pieces. The typical construction such a more complicated term -namely corresponding to two pieces along which the rule (2.10) is used. It would lead to a contribution to the Hilbert space for the second quantized even object given by a functional integral for $J$ 's (orח's) being fields on a two dimensional Euclidean manifold looking like

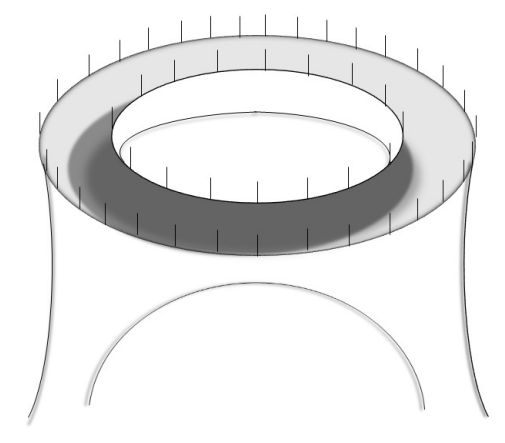

Figure 13: Topologically different one obtained by making use of two cones in the manner described in main text.

This drawing is meant to be obtainable topologically from the one for piece on which (2.10) were used only by taking a twodimensional piece of the "bottom" pull it up while it hangs together and then chop off the cap of the pull out. The piece of Hilbert vector 


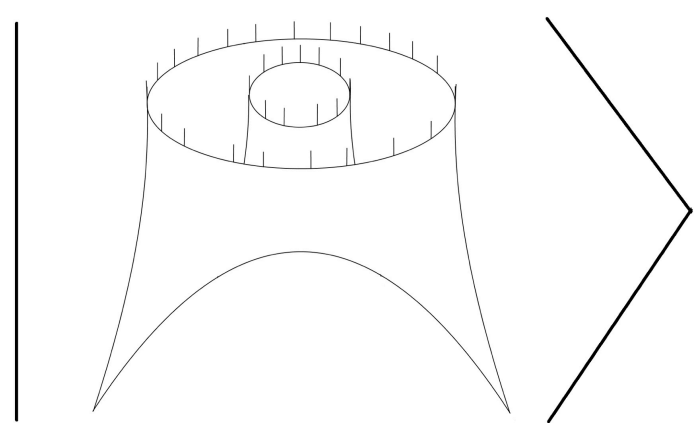

Figure 14: The Hilbert vector corresponding Figure ??, to be included when calculating S-matrix element.

has now two cyclic chains to be identified with corresponding ones in the final state if we shall use our $\mid$ string1, string2,inc. $\rangle$ to make an $S$-matrix element.

\section{An Encouraging Step;The Veneziano model except for the integration weight}

Having argued for functional -integral constructions as suggested by th above and with imaginary time, we have really obtained, what is usually considered the correct description of string development in a double description. The functional integrals are in our presetn philosophy just a mathematical trick to describe the wave function for the string states, while if you consider the functional integrals the usual way the surfaces drawn rather represent the development of the strings, even if in some way Wick rotated though. One shall note that in our philosophy there is in our object formulation no development. It is only a trick to construct the wave function. Nevertheless, there is an obvious similarity between the diagrams describing functional integrals which we obtain when we write down or describe the overlap between an initial $|i\rangle$ and a final $|f\rangle$ states in terms of the functional integrals and to the same $S$-matrix element corresponding development diagrams.

From this actually quite perfect correspondence we can in fact as a beginning derive that we must obtain the Veneziano[21] model(s) in question up to that an extra factor under the integration sign in the integral expression for the Veneziano model is more delicate to obtain. We believe that this inclusion of such pieces of cyclic chains cancelling each other so that no track of their existence is left in our description by means of objects can be of help to solve a problem, which we met in our development of our string field theory based on such object description:

The problem were the following: We sought to calculate from our novel string field theory the scattering of two particles into two others expecting to obtain a Veneziano model with three terms corresponding to the usual three pairs among the channeles s, t, and u. But ...

We got only one of these terms!

We did the calculation, that turned out in this way unsuccessfully in an infinite momentum frame "gauge"(=parametrization) choosing the right mover and the left mover coordinates ensuring a fixed amount of the "longitudinal" component of the 26-momentum for all objects.

Thus there were in this "gauge" choice no way to have the "longitudinal" component of momentum made opposite.

So there were no way in that "gauge" to realize the "Phantasy or cancellation pieces of cyclic chain". 
All cyclic chains corresponding to (open) strings would have to be "visible" in the second quantized object description.

But then two strings cannot become one by partial annihilation in the cyclic chain description .

In principle our "Novel String Field Theory" should just be a rewriting of a system of many strings interacting with each other. There should be nothing logically new, only reformulation! Whether we really have logical perfect correspondence (after quantization) depends, however, on how much information we count it that there is in our formalism. Strictly speaking we could make "philosophically different versions" of our model, each including different amounts of information in them. Only the one with large amount of information would match usual string theory. But we suggest to take the version with minimal amount of information most serious as our novel string field theory.

\section{The Classical Approximation Summary of "Layers of Existence Degrees"}

But in our formulation, the "Novel String field Theory" it is we think pedagogical to consider several layers of truth or existence corresponding to different versions with respect to including information into the formalism. Let us first describe these existence - "layers" in our classical (by classical formulation we have in mind that the single particle states are described classically with both $\Pi$ and $J$ having values simultaneously - in disagreement with Heisenberg uncertainty principle - while the objects are still second-quantized, so that a state in superposition of having different numbers of objects is in the picture) formalism, with which we started.

We have a series of steps from truly existing in our novel theory to being more and more phantasy, not really existing:

1. Fully existing the system of objects that can have both negative and positive energy - because they sit on a background of the "rough Dirac sea" (which is also fully existent, although we avoid having to go in detail formulating it.)

2. Chaining of Objects into Cyclical chains from the continuity of the strings and the boundary conditions we have the objects forming cyclically ordered chains with objects sitting with neighbor distances of the order of the "latticification cut off distance". Really we do not take it in our model that this chaining order has any physical existence in itself; but seeing a pattern of the "truly" existing objects with their $J^{\mu}$ 's and $\Pi^{\mu}$ 's we may let the nearness define for us a chaining. (A wrong way of chaining $\rightarrow$ bad continuity)

1. Fully existing

2. Chaining of Objects into Cycles

3. Pairing by this "pairing" we mean the information telling, which cyclically ordered chains together corresponds to a string. Open strings come from just one cyclically ordered chain each, while closed strings each need two, but here the total 26-momenta for the two shall be the same. So again knowing the cyclic chains there is some basis/restriction for guessing, which ones to combine. 
4. Cancelling pieces the phantasy pieces of opposite 26-momenta just invented.

As told: We first attempted a description with the single object -and also single string- being treated classically, but allowing quantum mechanics in the second quantization, so that we could make a superposition of even different numbers of objects or strings.

While in Thorn's bits from pieces of sigma have positions commuting with each other, the rightmover part of the position does NOT commute with itself. Rather there is for its derivative -which we want to work with a delta-prime function commutator.

If we shall have seperate creation and annihilation operators objects in any state, it would at least be a very unwanted complication if the degrees of freedom for one object and another one did not commute. Thinking classically on the single object state we should thus have zero Poisson bracket between the variables associated with two different objects. This is, however, impossible if we want the objects to represent at least the $\tau$-derivative of say the right mover part of the string position field $\dot{X}_{R}^{\mu}\left(\tau_{R}\right)$, because these rightmover fields or their derivatives for different values of the argument $\tau_{R}\left(\tau_{R}\right.$ is usually replaced by a complex variable $\bar{z}$ ) do not commute, thus do not have zero Poisson bracket.

Wanting

$$
J_{R}^{\mu}\left(\tau_{R}\right) \propto \dot{X}_{R}^{\mu}
$$

we define

$$
J_{R}^{\mu}\left(\text { for interval bit }\left[\tau_{R}-\Delta / 2, \tau_{R}+\Delta / 2\right]\right)=X_{R}\left(\tau_{R}-\Delta / 2\right)-X_{R}\left(\tau_{R}+\Delta / 2\right)
$$

where $\Delta$ is our cut off "length" into "bits" or "objects".

But these $\dot{X}_{R}^{\mu}$, s do not commute, but rather

$$
\left[\dot{X}_{R}^{\mu}\left(\tau_{R}\right), \dot{X}_{R}^{\mu}\left(\tau_{R}^{\prime}\right)\right]=i \delta^{\prime}\left(\tau_{R}^{\prime}-\tau\right) \neq 0
$$

Replacing the $\tau_{R}$ parametrization of right movers - replacing $\bar{z}$ which were complex - by a discrete counting $I$ taking integer values our discretized object variables

$$
J_{R}^{\mu}(I)=X_{R}^{\mu}\left(\tau_{R}(I)+\Delta / 2\right)-X_{R}^{\mu}\left(\tau_{R}(I)-\Delta / 2\right),
$$

where say $\tau_{R}(I)=$ constant $+I * a$, obey crudely at least that even $I$ object variables $J_{R}^{\mu}(I)$ commute with each other, and the odd ones commute with themselves, However the even ones do NOT commute with their TWO ODD neighbors!

This is just describing a discretized delta-prime function.

We dueled in constricting our SFT to only consider the even numbered objects as independent objects. Then we let the variables - especially $J_{R}$ for the ODD objects be written in terms of the conjugate variables $\Pi^{\mu}(I)$ of the neighboring even object variables:

$$
J_{R}^{\mu}(I)(\text { for odd } I)=-\alpha^{\prime} \pi\left(\Pi^{\mu}(I+1)-\Pi^{\mu}(I-1)\right) .
$$




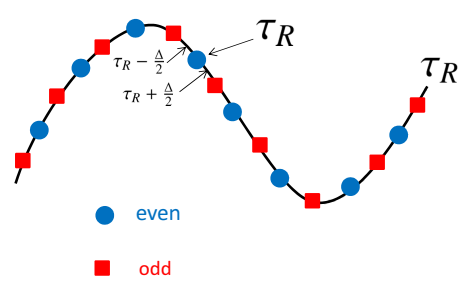

Figure 15: The string consists of a series of even and odd objects along the string alternatively put. As is depicted as an example the string is parametrized as the Figure 15.The right mover is parametrized by $\tau_{R}$. If the one even object is in $\tau_{R}$, the neighboring two objects are odd ones: one of them is situated at $\tau_{R}-\frac{\Delta}{2}$ and the other is at $\tau_{R}+\frac{\Delta}{2}$, where $\Delta$ is the cut off length into objects.

the $\Pi(I \pm 1)$ to be used here are numbered by the even numbers $I \pm 1$ and thus can be the conjugate momenta of the assigned $J_{R}^{\mu}(I \pm 1)$ to the even objects respectively.

We can consider this expression for the odd $J_{R}$ as a kind of "integrating up" the odd $J_{R}$ to construct/give us the $\Pi$ 's. For an even value of the integer $K$ we solve our prosed equation for the odd $J_{R}$ 's

$$
\Pi_{R}^{\mu}(K)=\frac{J_{R}^{\mu}(K-1)+J_{R}^{\mu}(K-3)+J_{R}^{\mu}(K-5)+\cdots}{-\alpha^{\prime} \pi} .
$$

It looks that with this "integrating up" information on the original continuum string variable $\dot{X}_{R}^{\mu}\left(\tau_{R}\right) \sim$ $J_{R}^{\mu}$ has been moved away in a non-local way for odd discrete points and is stored as the $\Pi_{R}^{\mu}$ for even argument.

It may be less serious though since $\dot{X}_{R}^{\mu}\left(\tau_{R}\right) \sim J_{R}^{\mu}$ were already differentiated, so really the $\Pi_{R}^{\mu}$ becomes essentially the right mover part of the position variable for the string.

If our so called string field theory is only a theory of essentially free massless objects, then it is a mystery: where is the string?

Do we even get the Veneziano model out of it? Yes we do. We actually can calculate to obtain Veneziano model -actually though for an OVERLAP between initial and final state rather than for 
a complicated S-matrix.

\section{Main idea in Calculating Veneziano Amplitude}

1. Since nothing goes on the S-matrix can only be unity and the S-matrix element just an overlap of in and outgoing states $\langle f \mid i\rangle$.

2. We write these in or outgoing states by having for each particle a wave function in terms of objects.

3. These wave functions are written by means of an IMAGINARY TIME functional integral for a STRING extracting the ground state (of the string) by it surviving long imaginary time development.

We wrote of the overlap becoming the scattering aplitude as the various pieces of surfaces with corresponding functional integrals, that were used to deliver the wave functions in object formulation corresponding to the incomming string, and to the outgoing ones. By looking at the various cases we see that for the

$$
1+2 \rightarrow 3+4
$$

scattering of ground-state (tachyons, in bosonic string theory), we realize at first that the combined surfaces modulo conformal and topology conserving transformations consists of an $S^{2}$-sphere with the four external ground state particles/string being attached to this $S^{2}$-sphere;

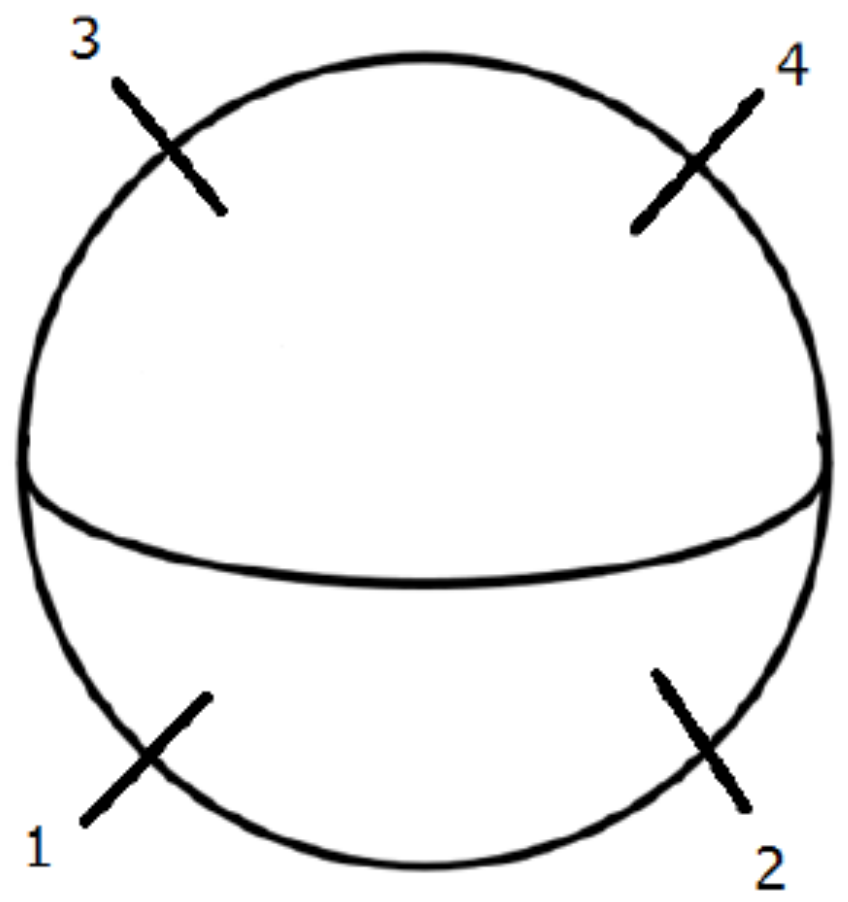


Figure 16: Illustration of four ground state scattering $1+2 \rightarrow 3+4$ where the surface is an $S^{2}$-sphere.

Because of the conformal invariance -up to an anomaly- of the funcitonal integral the possible inequivalent configurations of the four external lines on the Riemann-sphere $S^{2}$ is given by a single complex anharmonic ratio

$$
A=\frac{z_{1}-z_{3}}{z_{2}-z_{3}}: \frac{z_{1}-z_{4}}{z_{2}-z_{4}}
$$

where the $z_{i}$ 's are the Riemann surface notation places for the four external lines. By considering the construction in more detail one can see that in fact this anharmonic ratio $A$ becomes real and that all possible real values of $A$ can become realized. By the three values

$$
A=0,1, \infty
$$

corresponding to that a couple of external line attachments coincide the real axis compactified to the topology of an $S$ circle is divided into three intervals:

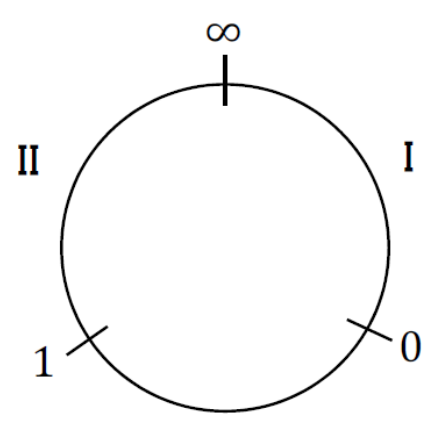

III

Figure 17: Inequivalent configurations of the four external lines on the Rieman-Sphere $S^{2}$ are $A=0,1, \infty$. The external line attachments is divided. $S^{2}$ topology's circle is divided into three intervals: I, II and III.

Each of these three pieces would, provided we obtain the right external- momentum- independentmeasure $\rho$ say, give rise to a term in the scattering amplitude as there are in the Veneziano model

$$
g^{2}(B(-a(t),-\alpha(s))+B(-\alpha(s),-\alpha(u))+B(-\alpha(u),-\alpha(t))) .
$$

For instance with an appropriate definition of an anharmonic ratio of the type of $A$, namely

$$
X_{\mu t}=\frac{z_{1}-z_{4}}{z_{1}-z_{2}}: \frac{z_{3}-z_{4}}{z_{3}-z_{2}}
$$

where we use the enumeration of external lines for $1+2 \rightarrow 3+4$ and call the $14-$ or $23-$ channel the $t$-channel

$$
\left(-p_{1}+p_{4}\right)^{2}=\left(-p_{2}+p_{3}\right)^{2}=t
$$

and the $13-$ or 24-channel the $u$-channel

$$
\left(-p_{1}+p_{3}\right)^{2}=\left(-p_{2}+p_{2}\right)^{2}=u
$$


we obtained in our previous article [] that we got such an extra measure $\rho$ that the amplitude piece coming from

$$
0 \leq X_{\mu t} \leq 1
$$

because

$$
\int_{0}^{1} X_{\mu t}^{-\alpha_{t}(t)^{-1}}\left(1-X_{\mu}\right)^{-X_{u}(u)^{-1}} \rho\left(X_{\mu t}\right) d \alpha_{\mu t}=B(-\alpha(t),-\alpha(u)) .
$$

Our hope is that, an evaluation of the external momentum independent factor $\rho$ for the two other regions in the anharmonic ratio, will turn out to deliver the two missing terms, $B(-\alpha(s),-\alpha(t))$ and $B(-\alpha(s),-\alpha(u))$.

We should stress that we have already by refering to usual string scattering theory that the factor in the integrand depending on the external momenta has the correct form for giving these missing terms. This is already extremely promissing for that we shall obtain the full Veneziano model.

Let us also stress that in fact the two missing terms come from those terms in the overlap which in the object representation have parts of cyclic chains corresponding to different, say incoming strings just compensating/annihilating each other. So the two [] missing terms in our previous paper only come about, because we now have -due to rough Dirac sea- (See Appendix A and B) the possibility of cyclic chain pieces with also negative energy.

\subsection{The simplest case of only positive $J^{+}=\frac{a \alpha^{\prime}}{2}$ (even) objects:}

Here as a starting example of calculating Veneziano model we consider the case that two incoming open strings have only postive $J^{+}=\frac{a \alpha^{\prime}}{2}$ objects in their cyclic chains, and also the two outgoing strings 3 and 4 have only positive $J^{+}$objects.

Then all the objects of the cyclic chain of string 1 must go to either 3 or 4 and the separation with fewest breakpoints (points where one class of objects stop and a new class begins) means that the objects on the cyclic chain of string 1 just falls into two pieces. One of these two pieces go into the final state string 3 , the other one goes into string 4 .

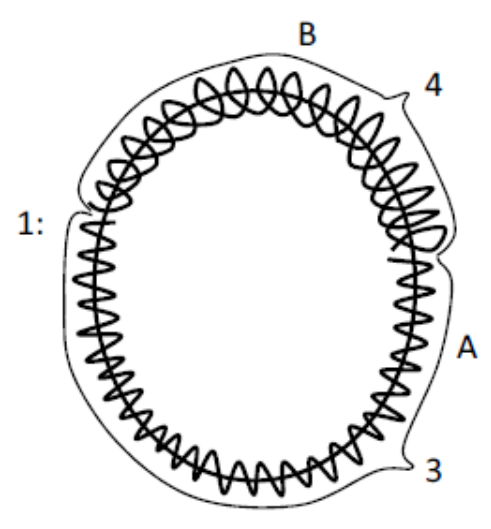

Figure 18: All the objects of the cyclic chain of string 1 must go to either 3 or 4 and the separation objects on the cyclic chain of string 1 fall into two pieces 3 or 4 . 
By saying the same for string 2 you soon end up with scheme fig. 19.

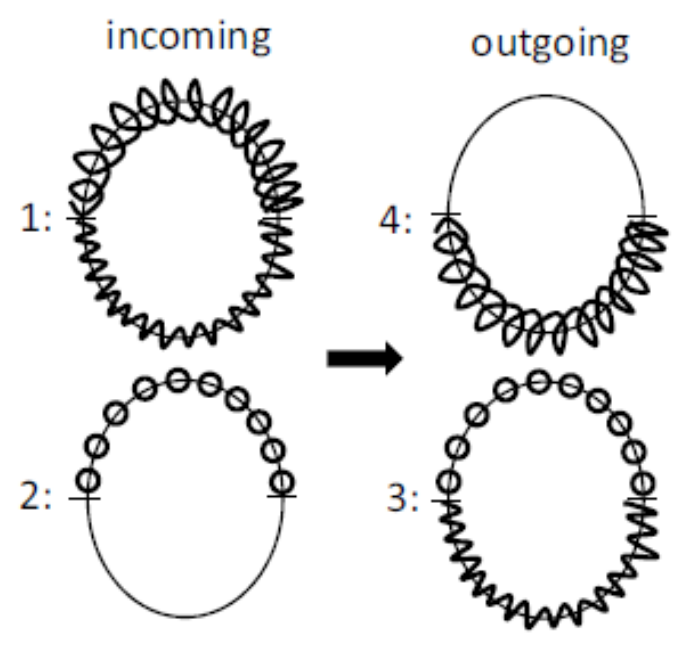

Figure 19: The figure illustrating how different of the tical state string 1 and 2 goes under the overlay sales lotion.

How many objects there are in the four different classes marked by their curve signatures

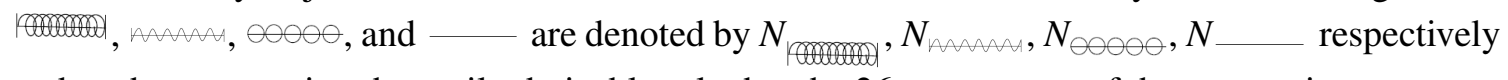
and we have assuming the easily derivable rule that the 26-momentum of the open string

$$
P_{\text {string }}^{\mu}=\frac{1}{2 \pi \alpha^{\prime}} \sum_{I=0}^{N-1} J^{\mu}(I)
$$

that the "longitudinal momenta" of the four strings (We use a light cone or metric notation with $\eta^{+}=\eta^{0}+\eta^{25}$ and $\eta^{-}=\eta^{0}-\eta^{25}$ )

$$
\begin{aligned}
P_{\text {string } 1}^{+}=\frac{a \alpha^{\prime}}{2 \Pi \alpha^{\prime} \cdot 2}\left(N_{1}\right) & =\frac{a}{4}\left(N_{1}+N_{1}\right) \\
P_{\text {string } 2}^{+} & =\frac{a}{4}(N+N \\
(*) P_{\text {string } 3}^{+} & =\frac{a}{4}\left(N_{\ominus 0000}+N\right. \\
P_{\text {string } 4}^{+} & =\frac{a}{4}(N+N
\end{aligned}
$$

Having in mind that we shall calculate the Veneziano amplitude for one set of external 26-momenta at a time, we must get the amplitude for one such fixed set of external momenta with a sum running

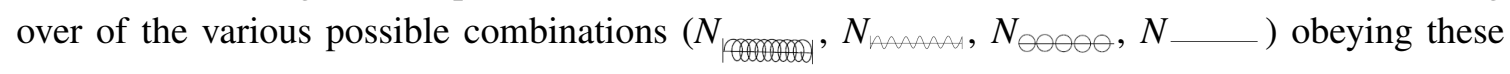
equations $(*)$ for the fixed external momenta. This means obviously that the variations under the summation obey

$$
\Delta N_{1}=-\Delta N_{\text {man }}=+\Delta N_{\odot 0000}=-\Delta N_{-}
$$


Thinking of the $N_{\text {, }}, N_{\text {and }}$ etc. as true numbers of objects, they cannot become negative.

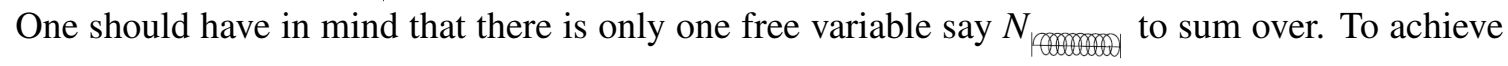
the most simple range over which to sum we would much like that $N_{\text {and }} N_{\odot 000 \ominus}$ would run down to 0 simultaneously and that $N_{\text {and }}$ and $N$ would also go to zero simultaneously, in the opposite end of the integration/summation so to speak. But from $(*)$ this wish would imply

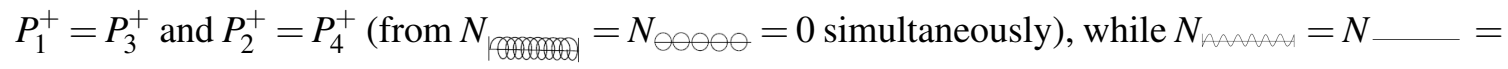
0 simultaneous imply $P_{1}^{+}=P_{4}^{+}$and $P_{2}=P_{3}$. All together these wish-requirements thus mean

$$
P_{1}^{+}=P_{2}^{+}=P_{3}^{+}=P_{4}^{+}
$$

If we trusted that the theory of ours were Lorentz invariant we might achieve this equality (??) by an appropriate Lorentz transformation.

If we do not have this case of equal $P^{+}$'s there can be different ways of connecting the objects from initial to final state and it may get more complicated.

Then under the assumption of the "longitudinal momenta" $P_{\kappa}^{+}$for $\kappa=1,2,3,4$ being all equal;

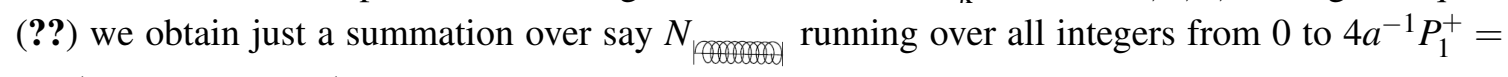
$4 a^{-1} P_{2}^{+}=\ldots=4 a^{-1} P_{4}^{+}$.

We shall approximate this summation by an integration

$$
\sum_{I \in\left[0,4 a^{-1} P_{1}^{+}\right]} \rightarrow \int d N
$$

and from here obtain the integration so characteristic for the Euler's Beta function form of cntributions to the Veneziano model.

A lot of details of the set up of the calculational procedure is to be found in []. The main point is that one first write the wave function for the set of even objects $-N_{\text {and }}+N_{\text {string }} / a$ of them, or rather only $2 P_{\text {string }} / a$ of them when we only want the even ones- by being given by the value of a functional integral being a regularized (cut off e.g. by a lattice) Feynman-Dirac-Wentzel one with the Nambu action or better one already made into a conformal gauge form

$$
\int \exp \left(-\left(2 \pi \alpha^{\prime}\right)^{-1} \int_{A}\left(\vec{\partial} \phi^{\mu}\left(\sigma^{1}, \sigma^{2}\right)\right) d \sigma^{1} d \sigma^{2}\right) D \phi^{\mu}
$$

(The index $\mu$ is the Lorentz index; but basically one develop the different factor with different $\mu$ separately; at the end multiply then.)

$A$ is chosen to be either a (unit) disk with the center punctured out or what is via conformal transformations a half infinite cylinder.

-By this conformal transformation the punctured center corresponds to the cylinder running to infinity end- 


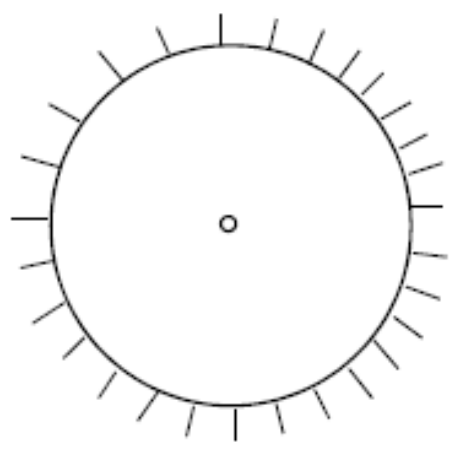

(a) A unit disk with the center punctured out

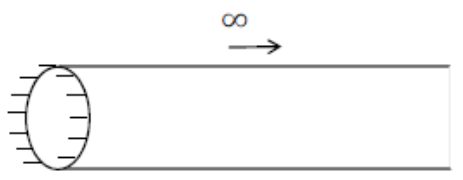

(b) A half infinite cylinder

Figure 20: (a) and (b) are conformally equivalent

Along the edge of the disk or conformally equivalent the finite end of the half infinite cylinder the objects in the cyclic chain corresponding to one of the strings, say string 1 , are imagined attached in a equidistant.

The meaning of this attachment to the edge is that the values $J^{\mu}(I)$ associated with objects around the cyclic chain are identified with differencec of neighboring values of the functional integral (dummy) variable $\phi^{\mu}$ (see equation (5.15). If there are in total $N$ objects in the cyclic chain the attached object $I$ may be at the angle -along the disk-edge- $\theta(I)=2 \pi \cdot \frac{I}{N}$ and then we put (say)

$$
J_{R}^{\mu}(I) \text { ident. } \phi^{\mu}\left(\exp \left(i 2 \pi \frac{I+1}{N}\right)\right)-\phi^{\mu}\left(\exp \left(i 2 \pi \frac{I-1}{N}\right)\right)
$$

for all even $I$. Because of our technique of only taking the even objects as truly existing and being directly used in the second quantized description in our Hilbert space we only use the even $I$ values here.

Here the complex numbers exp $i 2 \pi \frac{I \pm 1}{N}$ refers to that the disk-shaper region A for the functional integral is imbedded into the complex plane as the unit disc (and so the norm unity numbers lie on the edge of the disc.)

The reader can relatively easily see first that the functional integral comes to depend on the object $J^{\mu}$ variables in a "Gaussian" (exponential of a quadratic expression in these $J^{\mu}(I)^{\prime} s$ ) way and then secondly that the "fluctuations" in the various Founrier resolution coefficients of these $J^{\mu}(I)^{\prime} s$ as a function of the angle $2 \pi \frac{I}{N}$ become the same as these fluctuations as estimated e.g. in our article on the mass spectrum of the string in our object-formulation [2].

But the easiest way to see that the functional integral gives the wave function is by thinking of producing the ground state by propagating during an imaginary time span. If this time span is taken to be long, go to infinity, the dominantly surviving string state will be the ground state.

This is illustrated above by the figure 


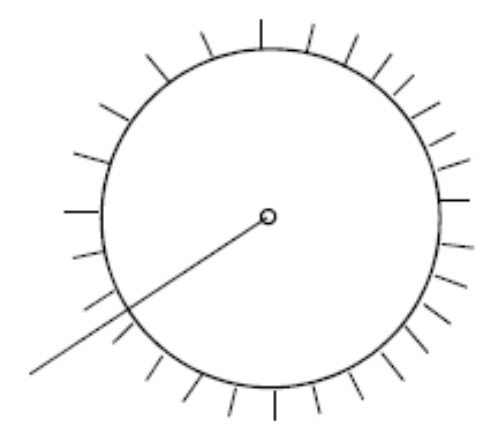

Figure 21: The ground state of the string state is obtained in the functional integral by propagating during an imaginary time span. If this time span is taken to be long, e.g. go to infinity, the dominantly surviving string state is the ground state.

Now we have four external particles giving rise to four such wave functions which can be written by means of discs or half-finite region functional integrals.

We shall mark the objects sitting along the edges of these discs by the symbols on fig.[21]. Having in mind that we can as well as a disc use the complement of a disc depicted as Figure 22.

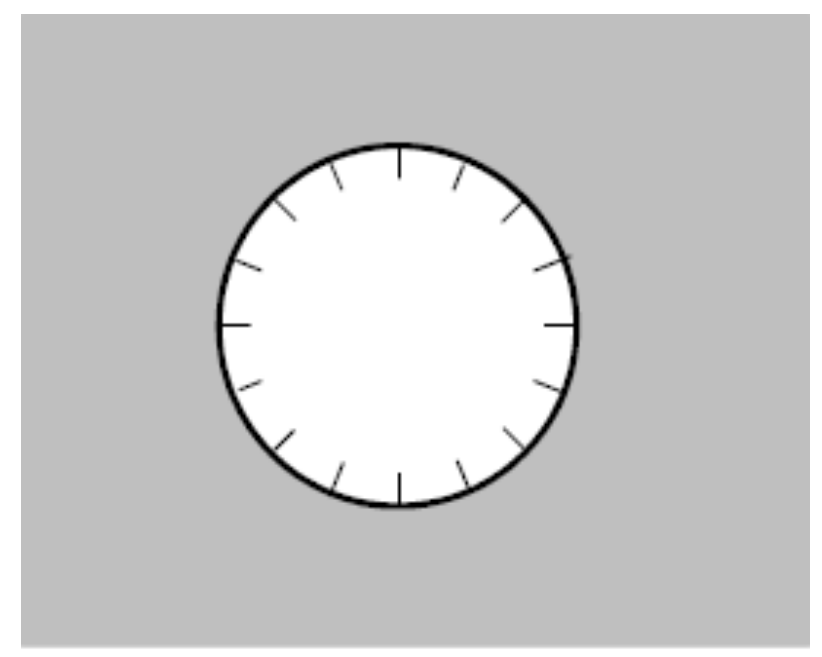

Figure 22: The complement of disc. Four external particles that give rise to four wave functions. They are written by means of discs or half-finite region functional integrals. The objects sit along the edges of the discs. We can use as well as disc's complement of a disc. In fact the region outside the disc is the two dimensional region. On this figure we have drawn two layers with the four discs put in as discs and complements. 
The meaning of the region is that outside a disc as the 2-dimensional region we can figure out a way to match the regions of objects marked with corresponding symbols, even in such a way that all four discs or complements lay on two layers in the complex plane/Riemann sphere. In fact we shall put them so that 1 and 2, i.e. the incoming particles/strings have their wave functions represented by (proper) discs -lying in the two layers respectively -while the outgoing strings 3 and 4 are represnted by complements of also unit discs lying correspondingly by in the two layers over the complex plane.

In this way we get the whole complex plane covered doubly, one representative in each layer all over.

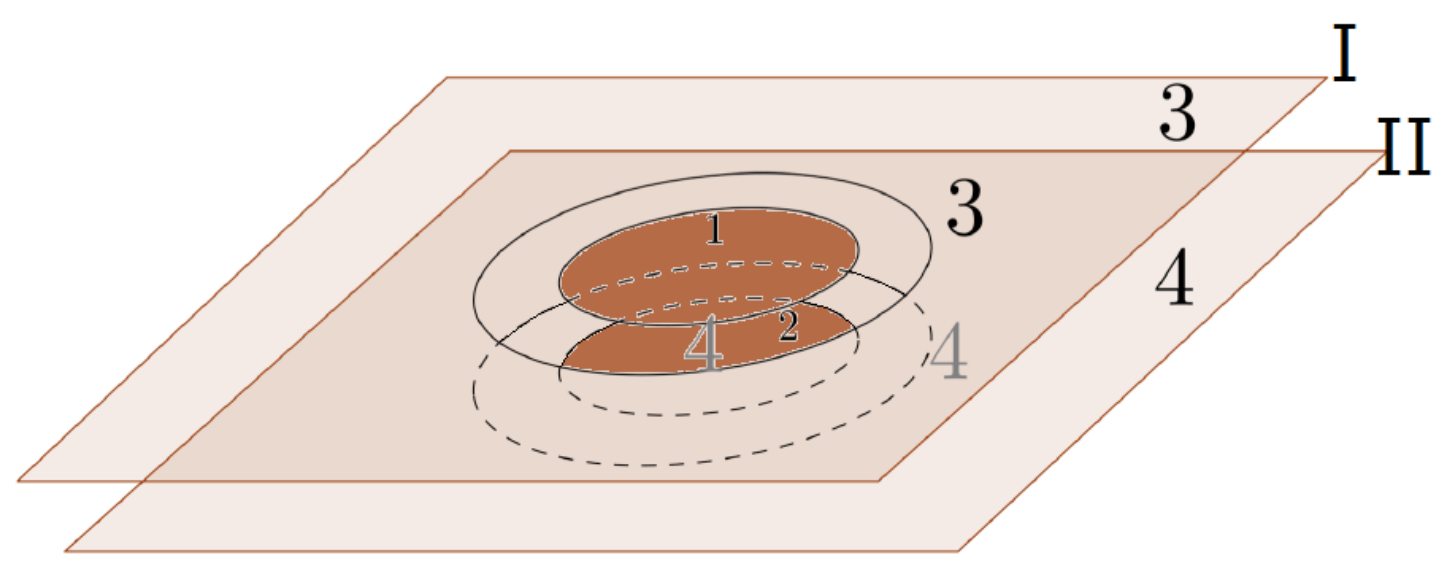

Figure 23: The two lines structure.

Fig.Caption: Layer first one is denoted I while the second is II. These two layers are connected.

To be definite we can choose to put in the first layer, called I, the disc for string 1 and the complement of the disc for string 3.

We put them so that the common edge for these two regions are just placed side by side (on the unit circle). Since by our assumption of all four strings having the same longitudinal momenta

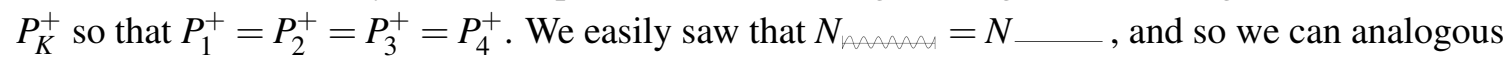
in the second layer, called II, put the pieces marked with _ in the edges for 2 and 4 just covering the same part of the unit circle (just now on layer II) as the piece marked connecting 1 and 3. 


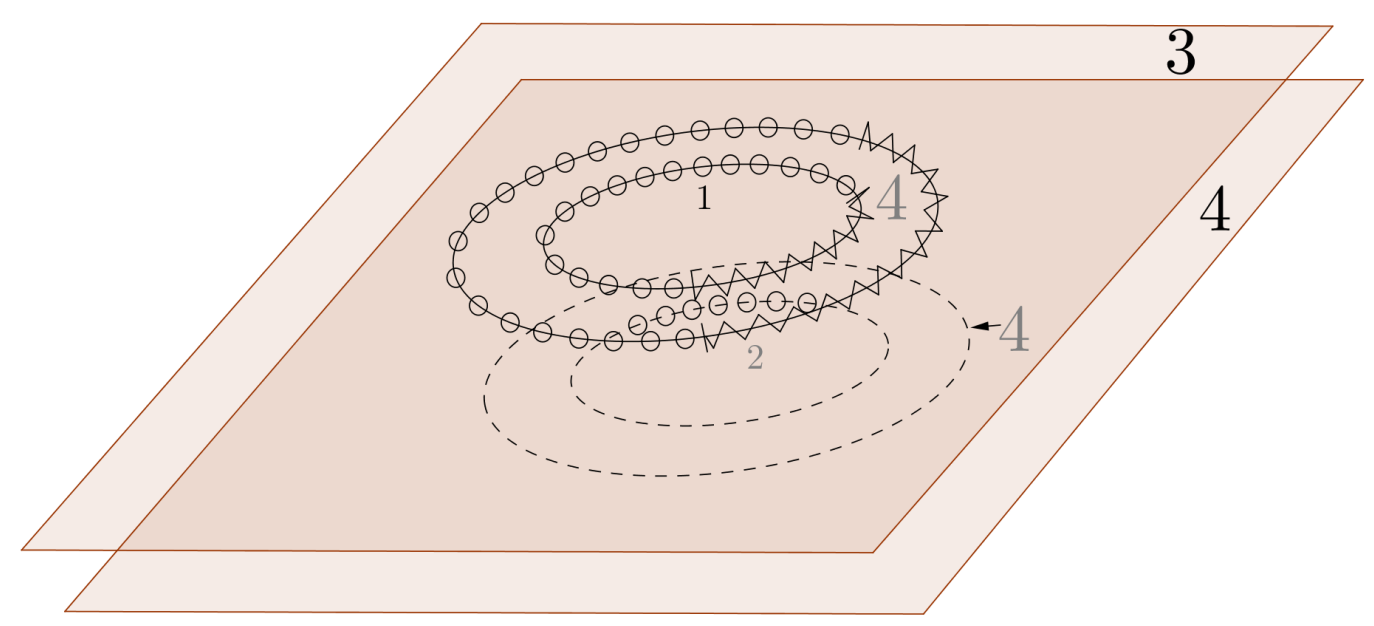

Figure 24:

Fig.Caption:Here we have drawn the markings ment 3 and along the pieces of edge for respectely 00000 on disc2 and on disc1.

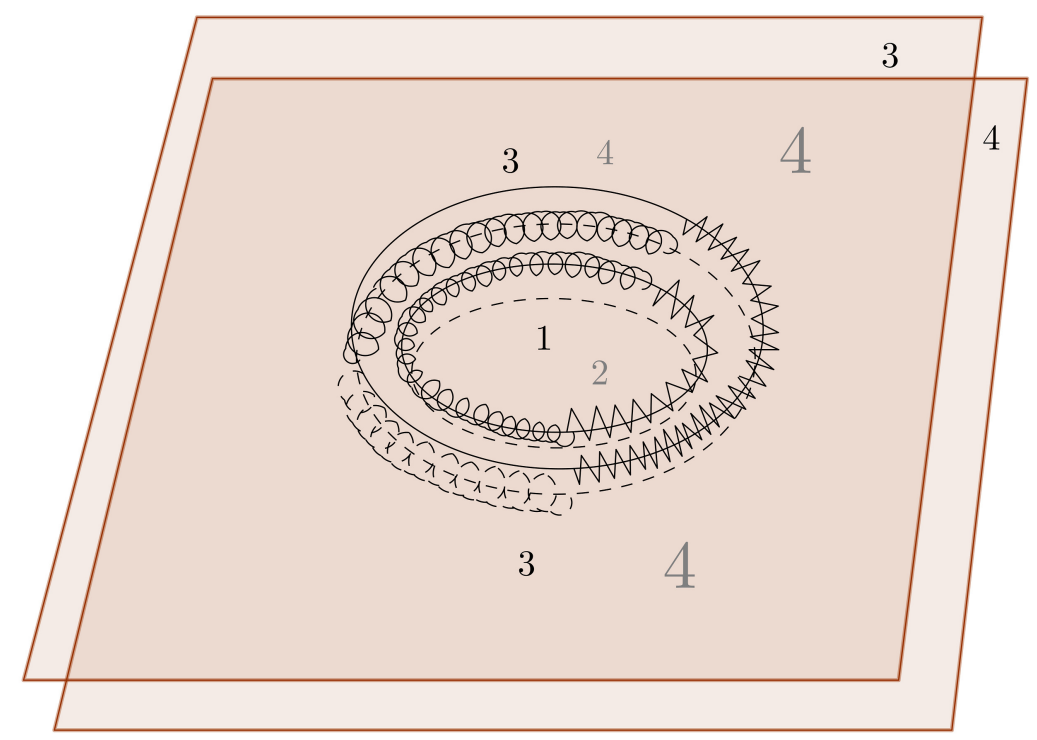

Figure 25:

Fig.Caption:On this fig. 25 we show in a similar way the edge- decorations on the disc 1 marked and the corresponding pieces of the edges of the complements 3 and 4 .

In this placement of these discs and complements of discs one easily sees that by a simple cut running along the remaining part of the unit circle, (a cut along which layer I is continued into I I and oppositely,) we achieve to continue disc for 1 into the complement of disc for 4 across the 
piece of unit circle now marked Analogously the disc for 2 gets continued along the edge across the cut into the complement of disc associated with string 3 .

The cut connecting I with II and oppositely must have a branch point in each of its two ends. Aboutwe manage to realize in the two-layered complex plane all the four gluings corresponding to objects in initial and final states being identified.

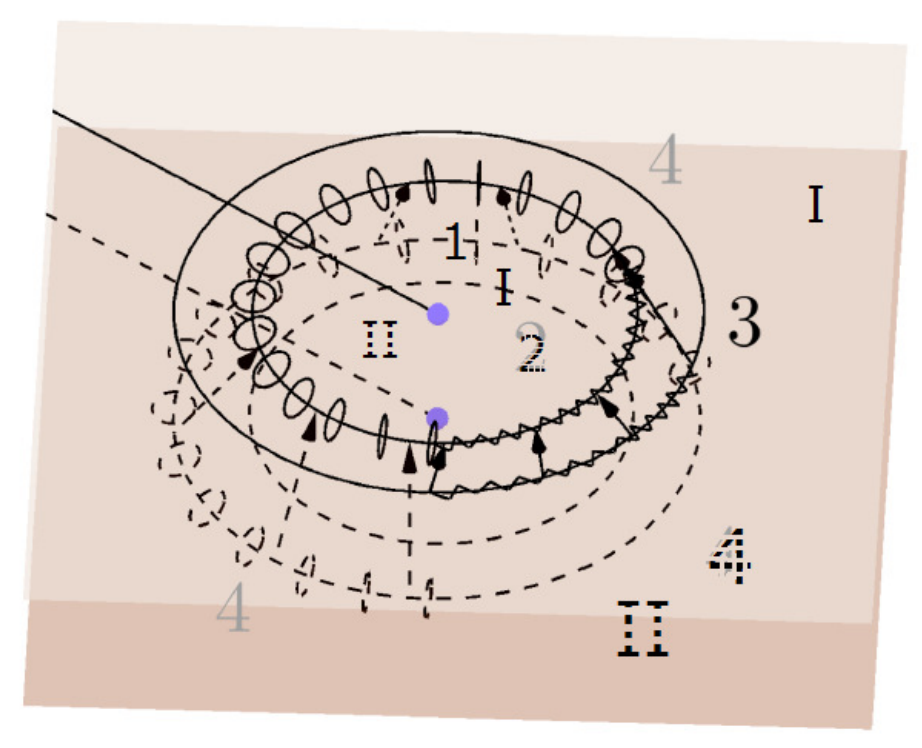

Figure 26:

Fig.Caption:On this figure we have drawn arrows to represent the gluings, but to avoid making the figure incomprehensively complicated we left out the arrows. That should have connected disc 2 with complement 3 by arrows crossing in between the arrows connecting disc 1 with complement 4 both marked 00000 . Similarly we left out the arrows that should have connected disc 2 and complement 4 both in the second layer II.

The gluing together of the regions for the functional along the curves where we identify objects in and outgoing states is supposed to lead to just the functional integral over the composed region (union of the glued together regions.)

The full functional integrals with boundary conditions from 26-momentum inlets in the centers of the discs or in the infinity in the case of the complement of a disc becomes the overlap under the specific way of identifying objects in the initial and the final state.

The full overlap is therefore a sum over all the ways of identifying the objects of the overlaps under the specific identificaitons.

But we have made the approximation of only considering significant the identification patterns with the lowest number of jumps, where neighboring objects do not follow each other into the next cyclic chain.

Now we shall to evaluate the functional integral for a given identification of objects make use of that 
modulo the anomaly -to which we shall return- the value of the functional integral is invariant under conformal transformations of the two-dimensional region associated with this functional integral.

The most important step in the calculation is now to by a conformal transformation map the double layered complex plane/or better Riemann sphere into a single layered one. Very suggestively this shall be done by a square root type of analytic function, because a square root ambiguity gives two possible values.

Then we can use a complex $z$ give two different result values $f\left(z_{I}\right)$ and $f\left(z_{I I}\right)$ depending on which layer I or II we imagine the complex number $z$ to be.

Layer I drawn full

Layer II drawn punctured

All the four unit circles really coincide. It is only drawing technique they differ. Edges of 1 and 3 to be identified are on layer I.

Now we shall seek to map our two layered representation of the two dimensional surface on which the functional integral is to be calculated by an analytical/conformal map into a one-layered one. To get inspired to what map $f$ say to choose we shall at first look at the situation near the two branch points. Imagining a litlw "circle" meaning a curve in a distance $\varepsilon$ ( $\varepsilon$ small) from the very branch point we see that it get actually the circumference $4 \pi \varepsilon$ rather as a usual circle having only $2 \pi \varepsilon$.

This implies that the function performing the map $f$ should have at square root singularity a square root like behavior near these two branch points.

Denoting the variable in the complex plane $z$ we get to each complex number $z$ two sheets with the two points on the full two-layered surface $z_{I}$ and $z_{I I}$. Here the $z_{I}$ is the point on sheet I and $z_{I I}$ the one on sheet II.

In this notation we can write down the attachment points $a_{i}(i=1,2,3,4)$ for the four external points:

$$
\begin{aligned}
& a_{1}=O_{I} \\
& a_{2}=O_{I I} \\
& a_{3}=\infty_{I} \\
& a_{4}=\infty_{I I} .
\end{aligned}
$$

Denoting the $z$-numbers corresponding to the branch points as $b_{A}$ and $b_{B}$ the need for square root behavior means that near these branch points we shall have

$$
f \approx \pm \sqrt{z-b_{i}} \quad i=A, B
$$

or

$$
f \approx \frac{1}{ \pm \sqrt{z-b_{i}}}
$$

Here as well as when we finally choose $f$ it is to be understood that the sign ambiguity is to be resolved differently on the two sheets I and II (for the same $z$ ). At other places singularities of $f$ should rather be avoided. Actually the reader may easily see that the function

$$
f(z)= \pm \sqrt{\frac{z-b_{A}}{z-b_{B}}}
$$


is a good proposal for the transformation and that with appropriate sheet dependence of the \pm we get indeed a mapping into a single layer all covering Riemann sphere(being the image of $f$ ). Indeed we could check that $f$ maps the two layered twodimensional region (for the functional integral) surjectively (i.e. onto) to the Riemann sphere (with variable $f$ ) by constructing the inverse i.e. 2 as function of $f$ : This inverse is gotten by the equation

$$
f^{2}=\frac{z-b_{A}}{z-b_{B}}
$$

meaning

$$
\left(b_{B}-z\right) f^{2}=z-b_{A} \quad \Longrightarrow \quad z=\frac{b_{B} f^{2}+b_{A}}{1+f^{2}}
$$

Under this map $f$ it is easily seen that the attachment points $a_{1}, a_{2}, a_{3}$ and $a_{4}$ are mapped into

$$
\begin{aligned}
& f\left(a_{1}\right)=\sqrt{\frac{b_{A}}{b_{B}}} ; f\left(a_{3}\right)=1 \\
& f\left(a_{2}\right)=\sqrt{\frac{b_{A}}{b_{B}}} ; f\left(a_{4}\right)=-1
\end{aligned}
$$

Remembering that the branch points lie on the unit circle in the $z$-plane

$$
\left|b_{A}\right|^{2}=\left|b_{B}\right|^{2}=1
$$

we see that indeed all four images of the attachment points are also on the unit circle (but now in the $f$-plane)

$$
\left|f\left(a_{i}\right)\right|^{2}=1 \text { for } i=1,2,3,4,
$$

Note immediately that such positions are just like Koba-Nielsen variables [23] for a four point Veneziano amplitude.

\subsection{The philosophy of counting}

The major purpose of these rather detailed calculations is to obtain the Veneziano amplitude correctly not only by having the right external momentum dependence but also has the correct form with respect to 26-momentum independent factors, only depending on the integration dummy. In order for such an ambition level to make sense we must start also from a well defined integration measure or rather we prefer to start from a well defined summation over a number specifying how many objects are common for some couple of external strings. But such a summation over a number of objects we have already developed above in section 5.1 especially(5.14).

We have a little freedom not yet used to orient the discs and the complements, which we can use to arrange that the two branch points $b_{A}$, and $b_{B}$ become each others complex conjugate. In fact we may take

$$
b_{A}=e^{i \delta} ; b_{B}=e^{-i \delta}
$$

with $\delta$ being a real angle. it is then easily seen that the number say

$$
N_{\text {arn }} \propto \delta
$$




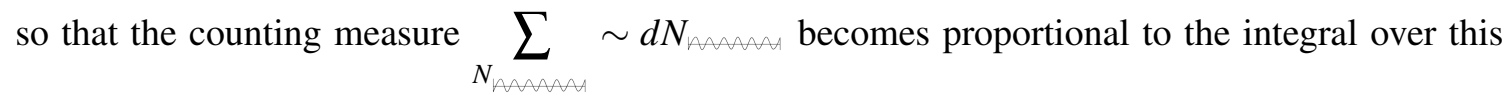
angle $\delta$

$$
\sum_{N_{\text {man }}} \sim d N_{\text {AnMA }} \propto d \delta
$$

\section{Evaluation of the integrand}

\subsection{Evaluation of the integrand}

Coming to the actual evaluation we must first face the problem that obtaining the wave function by a huge imaginary propagation leaves us with an arbitrary and divergent normalization. This divergence pops up by our need to led in at the attachment of the external particles/strings via small $\varepsilon$-radius discs.

Then the divergence shows up by the functional integral coming out in first approximation as the exponent of the classical action getting terms proportional to $\log \varepsilon$ into this classical action. However, since we shall not be so ambitious as to calculate the absolute normalization of the Veneziano amplitude it should be enough to just keep this $\varepsilon$ cut off the same for all the contribution to the Veneziano amplitude. Especially we should keep our $\varepsilon$ 's for the four external particle attachments fixed under the summation over the different numbers of objects exchanged say between 1 and 4 . That is to say we must keep the $\varepsilon$ 's constant while varying the integration variable $\delta$. The terms proportional to the $\log \varepsilon_{i}(i=1,2,3,4)$ are a priori expected to be also proportional to $p_{i}^{\mu} p_{\mu i}$, whereas terms involving the inner products of different external 26-momenta will be convergent. Taking these squares $p_{i}^{\mu} p_{\mu i}$ to be just the masses squared

$$
p_{i}^{\mu} p_{\mu i}=m_{i}^{2}
$$

and using that the mass of a mass-eigenvalue of a string state is just a constant, we see that these divergences $\propto \log \varepsilon_{i}$ are in principle not so severe because they only give constant factors to the over all amplitude, which we anyway give up calculating.

If we want to get the formula for the $B(-\alpha(t),-\alpha(u))$ written in terms of the usual integration variable

$$
B(-\alpha(t),-\alpha(u))=\int_{0}^{1} X^{-\alpha(u)-1}(1-X)^{-\alpha(t)-1} d X
$$

If we identify the channel $1+\overline{4} \rightarrow \overline{2}+3$ as the $t$-channel and $1+\overline{3} \rightarrow \overline{2}+4$ as the $u$-channel, while $1+2 \rightarrow 3+4$ is the $s$-channel, then the integration variable for this expression $\int X^{-\alpha(u)-1}(1-$ $X)^{-\alpha(t)-1} d X$ shall be the anharmonic ratio that goes to zero in the situation when the $u$-channel incoming $z$-variables approach each other. In fact we must take

$$
X=\frac{\left(z_{1}-z_{3}\right)\left(z_{2}-z_{4}\right)}{\left(z_{4}-z_{3}\right)\left(z_{2}-z_{1}\right)}=\frac{z_{1}-z_{3}}{z_{4}-z_{3}}: \frac{z_{1}-z_{2}}{z_{4}-z_{2}}
$$

where we have chosen the denominator so as to $X \rightarrow 1$ when the $z$-corresponding to say the incoming strings $1+\overline{4}$ approach each other.

In the analogue model terminology we imagine currents proportional to the 26 -momentum $p_{i}^{\mu}$ to 
be pumped in an $\varepsilon$-disc at the point $z_{i}$.

The external 26-momentum conservation will allow these currents to flow in a conserved way. The current running in at $z_{i}$ will if it just runs to infinity symmetrically by the in the model assumed specific resistance $2 \pi \alpha^{\prime}$ (we shall use $2 \pi \alpha^{\prime}$ rather than $\pi \alpha^{\prime}$ because we work with the double lead to a potential at the position $z$ in the Riemann sphere $\frac{1}{2 \pi} \cdot 2 \pi \alpha^{\prime} \ln \left|z-z_{i}\right|=\frac{\alpha^{\prime}}{2} \ln \left|z-z_{i}\right|$. Using this the total energy production rate in this analogue model would be

$$
\begin{aligned}
& \frac{1}{2} \sum_{\substack{i, j \\
i \neq j}} p_{i}^{\mu} p_{j \mu} \ln \left|z_{j}-z_{i}\right| \\
& \quad \text { (the } j=i \text { term diverge) }
\end{aligned}
$$

and so the exponential of this "heat production rate" becomes

$$
\prod_{i, j}\left(\frac{\left|z_{j}-z_{i}\right|}{\varepsilon_{i}}\right)^{-\alpha^{\prime} p_{i}^{\mu} p_{j \mu}} \cdot \prod N_{i}
$$

But these divergent factors although constant as functions of the external momenta are not constant as function of the $z_{i}$-variables of say as function of the anharmonic ratio $X$.

Remembering that the normalization factor for the $i$ th external particle is

$$
N_{i}=\varepsilon^{\alpha^{\prime} m_{i}^{2}}=\varepsilon^{\alpha^{\prime} p_{i}^{2}}
$$

and the 26-momentum conservation

$$
p_{1}^{\mu}+p_{2}^{\mu}=p_{3}^{\mu}+p_{4}^{\mu}
$$

or in an all ingoing notation

$$
p_{1}^{\mu}+p_{2}^{\mu}+p_{3}^{\mu}+p_{4}^{\mu}=0
$$

we recognize that $\varepsilon_{i}$ just appears to the power

$$
\text { "power of } \varepsilon_{i} "=-\alpha^{\prime} p_{i}^{\mu} \sum_{j \neq i} p_{j \mu}+\alpha^{\prime} p_{i}^{2}=0
$$

and thus there is really no dependence on these cut off $\varepsilon_{i}$.

From the definition

$$
X=\frac{z_{1}-z_{3}}{z_{4}-z_{3}}: \frac{z_{1}-z_{2}}{z_{4}-z_{2}}
$$

and we get

$$
\begin{aligned}
1-X & =\frac{\left(z_{4}-z_{3}\right)\left(z_{2}-z_{1}\right)-\left(z_{1}-z_{3}\right)\left(z_{2}-z_{4}\right)}{\left(z_{4}-z_{3}\right)\left(z_{2}-z_{1}\right)} \\
& =\frac{z_{4} z_{2}+z_{3} z_{1}-z_{1} z_{2}-z_{3} z_{4}}{\left(z_{4}-z_{3}\right)\left(z_{2}-z_{1}\right)} \\
& =\frac{\left(z_{3}-z_{2}\right)\left(z_{1}-z_{4}\right)}{\left(z_{4}-z_{3}\right)\left(z_{2}-z_{1}\right)}
\end{aligned}
$$


Using in the $p_{1}+p_{2}+p_{3}+p_{4}=0$ notation

$$
\begin{aligned}
s & =m_{1}^{2}+m_{2}^{2}+2 p_{1} \cdot p_{2} \\
& =m_{3}^{2}+m_{4}^{2}+2 p_{3} \cdot p_{4} \\
t & =m_{1}^{2}+m_{4}^{2}+2 p_{1} \cdot p_{4} \\
& =m_{2}^{2}+m_{3}^{2}+2 p_{3} \cdot p_{2} \\
u & =m_{1}^{2}+m_{3}^{2}+2 p_{1} \cdot p_{3} \\
& =m_{2}^{2}+m_{4}^{2}+2 p_{2} \cdot p_{4}
\end{aligned}
$$

and thus

$$
s+t+u=m_{1}^{2}+m_{2}^{2}+m_{3}^{2}+m_{4}^{2}
$$

to replace

$$
p_{1} \cdot p_{2}=\frac{1}{2}\left(m_{3}^{2}+m_{4}^{2}-t-u\right)
$$

We obtain

$$
\begin{aligned}
& \left(\prod N_{i}\right) \cdot \prod_{i, j}\left(\frac{\left|z_{i}-z_{j}\right|}{\varepsilon_{i}}\right)^{-\alpha^{\prime} p_{i}^{u} \cdot p_{j u}} \\
= & \prod_{\substack{i, j \\
i \neq j}}\left|z_{i}-z_{j}\right|^{-\alpha^{\prime} p_{i}^{u} \cdot p_{j u}} \cdot\left(\prod_{\substack{i, j \\
i \neq j}}\left|z_{i}-z_{j}\right|^{-\alpha^{\prime} m_{i}^{2}}\right) \\
= & \prod_{\substack{i, j)}}\left|z_{i}-z_{j}\right|^{-2 \alpha^{\prime} p_{i}^{u} p_{j u}-\alpha^{\prime}\left(m_{i}^{2}+m_{j}^{2}\right)}
\end{aligned}
$$

with $i \neq j$ but each $p$

$$
=\prod_{(i, j)}\left|z_{i}-z_{j}\right|^{\alpha^{\prime}\left(p_{i}-p_{j}\right)^{2}}
$$

on one order

$$
\begin{aligned}
& \cdot\left|z_{1}-z_{3}\right|^{-\alpha^{\prime} u} \\
& \cdot\left|z_{2}-z_{4}\right|^{-\alpha^{\prime} u} \\
& \cdot\left|z_{1}-z_{2}\right|^{-\alpha^{\prime}\left(-t-u+m_{3}^{2}+m_{4}^{2}\right)} \\
& \cdot\left|z_{3}-z_{4}\right|^{-\alpha^{\prime}\left(-t-u+m_{1}^{2}+m_{2}^{2}\right)} \\
& \cdot\left|z_{1}-z_{4}\right|^{-\alpha^{\prime} t} \\
& \cdot\left|z_{2}-z_{3}\right|^{-\alpha^{\prime} t} \\
& =X^{-\alpha^{\prime} u}(1-X)^{-\alpha^{\prime} t}\left|z_{3}-z_{4}\right|^{-\alpha^{\prime}\left(m_{1}^{2}+m_{2}^{2}\right)} \cdot\left|z_{1}-z_{2}\right|^{-\alpha^{\prime}\left(m_{3}^{2}+m_{4}^{2}\right)}
\end{aligned}
$$

\subsection{What is required to finish Veneziano model?}

To get the last bit of the way to obtain all three terms in the four point Veneziano model in our object scheme we have to obtain a definite counting of the number of ways of identifying or better 
bringing in correspondence the objects in the initial state with those in the finial state.

In our previous article we used as a combined gauge or parameter $\tau_{R}$ choice and discretization to impose the condition that each object has it $J^{+}$, a special component of its $J^{\mu}$ take a specific value

$$
J^{+}=\frac{a \alpha^{\prime}}{2}
$$

for all the objects.

This "longitudinal momentum" (or "longitudinal $J^{\mu}$ ") $J^{+}$(which is essentially the + component of momentum of the object) is defined

$$
J^{+}=J^{0}+J^{25}
$$

where $J^{25}$ is the infinite momentum frame direction. If, and that is indeed true since classically

$$
\left(J^{\mu}\right)^{2}=0
$$

the $J^{\mu}$ is "on shell" as a momentum then

$$
J^{+} \geq 0
$$

But if we allow "energy" $J^{0}$ to be negative then also $J^{+}$is not guaranteed to be positive.

As long as the $J^{+}$'s are guaranteed positive as we used in previous paper, then one can devide the positive +-momentum into $J^{+}$'s all being some given positive number

$$
J^{+}(I)=\frac{a \alpha^{\prime}}{2} .
$$

But now in order to be allowed to hope for obtaining all the 3 terms in the full Veneziano amplitude we must accept negative $J^{+}$'s.

So we must choose a gauge or parametrization and discretization choice that is more liberal with respect to allowing negative $J^{+}$too. The obvious suggestion is that in constructing the dicretization we first imagine dividing the cyclic chians corresponding to the strings into pieces with negative $J^{+}$'s and pieces with positive $J^{+}$'s. In order that this shall be nice it should be so that the $\dot{X}_{R}^{\mu}\left(\tau_{R}\right)$ that is proportional/essentially the same as $J^{\mu}$ is so smooth that the sign of $\dot{X}_{R}^{+}\left(\tau_{R}\right)$

$$
\operatorname{sign}\left\{\dot{X}_{R}^{+}\left(\tau_{R}\right)\right\}
$$

is constant over intervals of reasonably large size.

With some -may be a bit vague- continuity assumption these large intervals of fixed signs $\operatorname{sign}\left\{\dot{X}_{R}^{+}\left(\tau_{R}\right)\right\}$ is justified. Also one has in this spirit also the assumption that the pieces of a cyclic chain in an initial state string going in the overlap into a given final state cyclic chain will consist dominantly of very few pieces. It shall with highest weight be connected to one connected piece only. In the scattering the negative energy $J^{0}$ or negative $J^{+}$pieces have to for say an initial cyclic chain must either annihilate with positive piece in another incoming cyclic chain or go on as a negative piece in the final state. 


\section{Mixing of positive and negative objects}

Let us develop a bit our picture involving both "negative" and "positive" objects, by arguing that most likely the "positive" and "negative" objects will be severely mixed up. By this we mean that if we have chosen a gauge so that the various objects have their $p^{+}$or letter $J^{+}$taking the value either

$$
J^{+}= \pm \frac{a \alpha^{\prime}}{2}
$$

then quantum fluctuations in a typical string state will let the signs + or - to be assigned to the successive objects along the cyclically ordered chain fluctuate up and down, and there would be almost equal density of + and - along the chain. Having such fluctuations in mind we see that it would be wrong to think of the sign + or - being constant over long pieces. Rather we must think of sign taking both + and - many times inside even rather small pieces of the cyclically ordered chains.

We shall therefore not use the sign + or - to split up the cyclically ordered chain but rather only use a splitting according to with which of the other cyclically ordered chains (related of course to the other external strings/particles) the piece is annihilated or makes transition into.

We could for instance to keep simplicity in the sense of only considering the lowest number of pieces (in play) take the cyclically ordered chain for the open string number 1 as split into two pieces, one of which is annihilated with a "corresponding" piece in string 2, while the other piece (= the rest) goes on and becomes a piece in the outgoing string 4 . Of course in order that a piece from 1 can annihilate with one from the other incoming string 2 all the objects in the piece from string 1 must have just opposite sign + or - to the corresponding object in the string 2 piece.

This is a restriction, but with + and - about equally copious it is very analogous to that the signs in the piece in string 1 being incorporated in string 4 object for object must have the same sign + or -.

In our previous work[19] - in which we only used "positive $J^{+}$" objects - we restricted ourselves to a very special (type of) Lorentz frame in which all the four external strings had the same $p^{+}$and thus the same number of objects. When we now want to work with both positive and negative $J^{+}{ }^{\prime}$ s there would be no longer such a simple way by just coordinate choice - to make the four strings have equally many objects in them. However, we might still wave our hand very abstractly and suggest that there might still be degrees of freedom enough in e.g. choosing the frame to obtain by some - rather mysterious - choice the number of objects in the four different external strings to the same.

This is a simple choice and has the advantage of allowing only one way topologically ordered chains. However we avoid having more than one term to calculate for each of the three Veneziano model terms we go for.

Without having any clear proceeding let us though make the assumption that by some possible adjustment it is achieved that each of the four external particle/strings in the scattering $2 \rightarrow 2$ considered have equally many $N$ objects in their cyclically ordered chains. In this number counting we have counted both positive and negative $J^{+}$objects together and so there is no simple relation 
to the external momenta due to this choice.

Each of the four cyclically ordered chains we now represented by four circles - two incoming 1 and 2 and two outgoing 3 and 4 - with then pieces marked with various signatures such as Going for the simple diagram we only split each of the four cyclically ordered chains into two pieces. In our first calculation these pieces in 1 and 2 could only go on to string 3 or string 4, but now with negative $J^{+}$objects too we have the possibility that a series of objects in 2 annihilates with a corresponding series in 1. Similarly one could also get a couple of compensating/corresponding series in 3 and in 4 appear out of nothing so to speak. In the simplest case we have 4 pieces/series, which must occur is pieces of two different (external) strings each. Keeping the numbers of objects in all the four external strings fixed - and in fact for simplicity postulate them to all 4 have the same number of objects $\mathrm{N}$ - one immediate sees that varying the number of objects on say the series of objects in 1 annihilating with a corresponding series in 2 all the four pairs of series have to vary correspondingly. In fact the rest of the cyclically ordered chain for string 1 must decrease in number of objects by the same amount as the mentioned series increase. Of course the number of objects in 2 to annihilate with the mentioned series must have the same number of objects and thus it must also vary in the same way. Further arguing in this way we soon find that:

1) The series annihilating between 1 and 2 and the series annihilating between 3 and 4 follow each other, are in fact forced to be equal in numbers of objects, while

2) The series, that continue from 1 to 4 , and the one that continues from 2 to 3 must vary decreasing, when the ones under 2) increase in number of objects.

The reader may have in mind that it is the summation over all the different related lengths/number of objects, which these series can take on, that comes to correspond to the integration contained in the Eulers beta function which at the end is (the term in) the Veneziano model.

It is important that we take it that for each of the natural numbers in say, the annihilating pairs we get a contribution to the total overlap $\langle 3+4 \mid 1+2\rangle$, which we indeed identify with the scattering amplitude. Each of these contributions corresponding to a definite natural number of objects being annihilated between e. g. 1 and 2 is by our technique of writing wave functions for strings in terms of objects rewritten as a functional integral.

This functional integral we should remember is a functional integral obtained by composing functional integral - one for each external string - used to represent the ground state (tachyon state) for the external strings. Now the technique for calculating the important factor in such functional integrals consists in using the important property that "naively" these functional integrals are invariant under conformal transformation of the 2 - dimensional region over which the field over which one functionally integrates $\mathscr{D} \phi$ is defined. When we say that this conformal symmetry is only there "naively" it is because there is an anomaly contribution (correction to logarithm of a functional integral like the ones, we consider). Taking the limit of high density along the cyclically ordered chains - really the continuous string limit - the sum over the various natural numbers of 
objects in the piece used for specifying of course gets replaced by an integral over this number. With a certain permutation of the external particles/strings it turns out that the present integral over functional integrals becomes just the same as the one to which we got in our previous work [19]. By noticing this we can indeed immediately see that we obtain again an Euler beta function just now it is the term in the full Veneziano amplitude corresponding to there being resonances in the $t$ and the $\mathrm{s}$ channels (in the notation that $1+\overline{4} \rightarrow \overline{2}+3$ is the $\mathrm{t}$ - channel and of course $1+2 \rightarrow 3+4$ is the $\mathrm{s}$ - channel.). The previous work gave - we remind us - the contribution with resonances in the $u$ and the $t$ channels.

Although we hereby basically finished deriving the full Veneziano amplitude (since the third of the three terms needed in the full amplitude is of course completely analogous to the present one, just permuting say $1 \leftrightarrow 2$.), we might as an exercise remind ourselves about how we in the present $t$ and $\mathrm{s}$ - channel contribution put together the discs or half infinite cylinders used to provide the wave functions for the external particles (assumed to be in the ground state/or tachyonic state.). The way we have in the present example chosen the pieces to annihilate or to go on is so that there in no piece from 1 going on in 3 and no piece from 2 going on in 4 . To make a natural gluing together it is profitable that when say a piece goes on from 1 to 4 , then if 1 has its region for functional integration represented as (the unit) disc then 4 should have its represented as a coset of the unit disc, "a codisc".

In fact we must use the rule that when two external strings have a couple of corresponding pieces, meaning that they either annihilate or one goes on into the other one, then the regions for such two strings shall be represented differently with respect to disc versus "codisc". Thus if say, we take string 1 to have a disc both string 4 and string 2 must have codiscs. That is to say that the regions for the functional integral giving the wave function for string 4 and string 2 must be represented (by help of a conformal transformation) as cosets of the unit disc. But since string 1 and string 3 have no corresponding pieces it is o. k. to let string 3 obtain the unit disc again even though string 1 also has its region represented by the unit disc. Now we should note that the whole Riemann sphere is getting covered just twice. We namely have the string 1 and the string 3 regions both covering the same unit sphere, while the regions for string 2 and string 4 both cover the same coset of the unit sphere.

We use in other words a two layered cover of the complex plane (with the infinity point added.). Now it is of course crucial that the way these layers are to be connected along the unit circle has to be contemplated a bit. When for instance the string 1 cyclically ordered chain has a piece going on which becoming a "corresponding" piece in the cyclically ordered chain for string 4 , then this corresponds to that the layers must be contracted so that there is piece along the unit circle with an angular length proportional to the number of objects in piece going on 1 from to 4 along which now the sheet of 1 and the sheet of 4 are connected. This requirement that the sheets of 4 and 1 are connected along such a piece along such a piece along the unit circle means that the functions over which we functionally integrate go continuously and analytically across the unit circle along the piece of the specked length from sheet of 1 to sheet of 4 .

For the annihilation of a piece in 1 with one in 2 we have to require analogously that along a piece of the unit circle of angular length proportional to the number of objects in the annihilating 
pieces (which have of course equally many objects) the sheet for string 1 and the sheet for string 2 continue analytically and smoothly into each other, across the unit circle. Again there has to be similarly a smooth and analytical going across the unit circle from the sheet of 3 to the sheet of 4 . Actually the number of objects corresponding to the pieces of pair annihilating between 3 and 4 was seen above to be under our assumption just equal to the number in the piece pair annihilating between 1 and 2 .

Therefore we can actually arrange - or must arrange in fact - that along the same part of the unit circle the two layers - of the double layer covered Riemann sphere - both go smoothly and analytically through in the one layer considered as from 1 to 2 , while in the other layer it is from 3 to 4 . Along the rest of the unit circle, however, there should now be a cut meaning that now you rather have the smooth and analytical going through from one layer to the other one. In fact we namely along this "rest" of the unit circle have to have the sheet with 1 in the unit disc continue into the sheet with 4 in the "codisc", while the string 3 region in the disc continues into the string 2 region in the codisc.

This is easily seen to impose that we must have a cut here on this rest of the unit circle. By reassigning say the layers in the codisc region we could cause easily change the cut to be at the other piece of the unit circle. But there has to be one cut along the unit circle, and thus a couple of branch points in the two ends of this cut. We may call the branch points $b_{A}$ and $b_{B}$.

The figure means the complex plane and it is meant to have in mind that we consider two layers I and II so that a function $\phi$ - or if have in mind that there is one for each of the $25+1$ dimensions in Minkowski space except that one may in the gauge choice decide to treat a couple of them differently - a set of functions $\phi^{\mu}$ are really not only a function of the complex variable $z$ but also of the sheet number, which can be I or II. There is no cut $\phi^{\mu}\left(z_{I}\right)$ and $\phi^{\mu}\left(z_{I I}\right)$ are just separately analytical in the "classical approximation", while along the cut you have instead

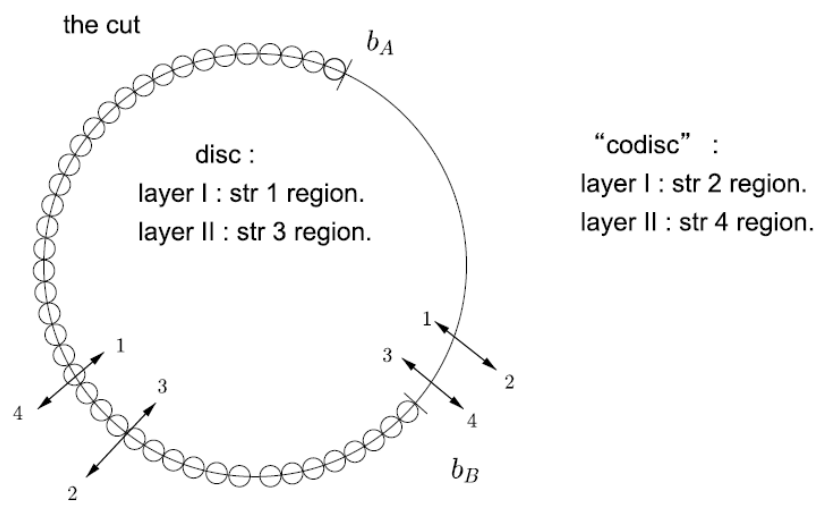

Figure 7.1

$$
\lim _{\left|z^{\prime}\right| \rightarrow|z|^{-}} \phi^{\mu}\left(z_{\mathrm{I}}^{\prime}\right)=\lim _{\left|z^{\prime \prime}\right| \rightarrow|z|^{+}} \phi^{\mu}\left(z_{\mathrm{II}}^{\prime \prime}\right)
$$


and

$$
\lim _{\left|z^{\prime}\right| \rightarrow|z|^{-}} \phi^{\mu}\left(z_{\mathrm{II}}^{\prime}\right)=\lim _{\left|z^{\prime \prime}\right| \rightarrow|z|^{+}} \phi^{\mu}\left(z_{\perp}^{\prime \prime}\right) .
$$

The symbols such as $1 \leftrightarrow 4$ crossing the cut mean that across that part of the unit circle the regions for string 1 and for string 4 get connected/get analytically into each other.

The cross in the center of the unit circle is to remind us that there is actual what we call an "inlet", one on each sheet just at $z=0$. There are in addition an "inlet" on each of the two sheets at $z=\infty$.

Since we can rotate the whole complex plane - the rotation just being a conformal from formation we can, if we like, arrange to have the two branch points being each others complex conjugate, say

$$
b_{A}=b_{B}^{*}=e^{i \delta} .
$$

Then the angle $\delta$ is actually proportional to the number of objects in the pieces that get annihilated. Our assumption of equally many objects in all of the four external particles has actually led to that there are equally many objects in the pair of pieces annihilating between strings 1 and 2 as in the pair of pieces annihilating between 3 and 4 . In terms of the integration to replace in the continuum limit the summation over different numbers of objects in the pieces the integration measure over which to integrate the functional integral becomes

$$
\text { "integration measure" } \propto d \delta
$$

and the integration region becomes

$$
0 \leq \delta \leq \pi
$$

Of course if one thinks instead of the length of the piece of the unit circle circumference between the two cuts $2 \delta$ the range becomes

$$
0 \leq 2 \delta \leq 2 \pi
$$

That is to say the integration will run from the two saddle points being infinitesimal small distance from each other and through at first increasing distance along the unit circle until the two branch points meet each other from the opposite side.

Now we have to remember that one can consider it that $25+1$ momentum flow along the surface which is now pictured - after some (mild) conformal transformation - as the double layer/sheeted Riemann sphere, and that the flux of $25+1$ momentum locally is given by the gradients of $\phi^{\mu}$ w.r.t. $\operatorname{Re} z$ and $\operatorname{Im} z$. The precise way to connect the gradient of $\phi^{\mu}$ with momentum flow can actually by chosen in a couple of different ways. To not go into a too complicated discussion we shall remind the reader that the result of the functional integration can be thought of in terms of the old analogy model of D.Fairlie and one of us (H.B.N), "the analogue model".[24] This formulation means that the functional integral gets expressed as the exponential of a certain heat production, or rather as the product of the exponentials of various heat productions, namely one for each dimension. For each spatial dimension the exponential is to be taken as minus the heat production while for the 
time dimension (if we have not be a gauge choice dispensed with it in a different way) we take truly the exponential of the heat production itself, i.e. with plus sign.

These heat productions are the ones calculated for the heat produced in a two dimensional conducting layer of the shape in our case doubly layered Riemann sphere on which the function $\phi^{\mu}$ is defined when the current is identified with the flow of momentum $p^{\mu}$.

For this flow of momentum and thereafter this heat production to be determine, it is of course crucial to know the boundary conditions about how much and where on the complex manifold on which $\phi^{\mu}$ is defined the momentum comes in or goes out. But when we constructed the half cylinders to provide us with the wave functions in terms of objects for the external particles we had of course to lead in from the infinite side the momentum for the external string state in question. By the conformal transformation of these half infinite cylinders to either discs or cosets of discs the inlet point where the external momentum shall appear/be let in becomes respectively $z=0$ and $z=\infty$. We therefore end up with the four "inlets" or inlet points in the complex manifold to be 2 at $z=0$ : (one with momentum $p^{\mu}$, on the region for string 1 of course, meaning on layer I, the other $p_{3}^{\mu}$ on layer II and 2 at $z=\infty$, namely $p_{2}^{\mu}$ on layer I and $p_{4}^{\mu}$ on layer II).

Summarizing a bit we reached to that we shall calculate the heat productions - one for each dimension - in a two dimensional conducting sheet of shape as the complex manifold described as a two layered cover of the Riemann surface and with a cut running between $e^{-i \delta}$ and $e^{i \delta}$ (say along a piece of the unit circle). The heat productions have to be calculated using the boundary conditions that currents of momentum $p_{1}^{\mu}, p_{2}^{\mu}, p_{3}^{\mu}, p_{4}^{\mu}$ are led into the two dimensional conducting sheet at the four described "inlet" points at

$$
\begin{aligned}
& z_{I}=0_{I}: p_{1}^{\mu} \\
& z_{\text {II }}=0_{I I}: p_{3}^{\mu} \\
& z_{I}=\infty_{I}: p_{2}^{\mu} \\
& z_{I I}=\infty_{I I}: p_{4}^{\mu} .
\end{aligned}
$$

To now calculate these heat productions we shall apply the essential (i.e. apart from the anomaly) conformal invariance and transform the two-layered complex manifold by a conformal transformation into an only single layered cover of the Riemann surface.

The transformation needed must just have square root singularities at the "branch-points" $b_{A}=$ $e^{i \delta}$ and $b_{B}=e^{-i \delta}$. In fact we define the transformation

$$
f= \pm \sqrt{\frac{z-e^{i \delta}}{z-e^{-i \delta}}}
$$

where the square roots usual sign ambiguity \pm has to be made into a dependence on whether the sheet is I or II.

Important for our final evaluation of the functional integrals is it to know which $f$-values $\left(f \in C^{\prime}\right)$ corresponds to the four "inlets". Trivial-except for considerations of how the \pm is chosen 
depending on the sheet I or II - calculation, insertion in gives :

$$
\begin{aligned}
& " p_{1}^{\mu} \text {-inlet" }: z_{I}=0_{I} \longrightarrow f_{1}= \pm e^{i \delta} \underset{\text { taken }}{=} e^{i \delta} \\
& " p_{2}^{\mu} \text {-inlet" }: z_{I}=\infty_{I} \longrightarrow f_{2}= \pm 1 \underset{\text { taken }}{=} 1 \\
& " p_{3}^{\mu}-\text { inlet" }: z_{I}=0_{I I} \longrightarrow f_{3}= \pm e^{i \delta} \underset{\text { taken }}{=}-e^{i \delta} \\
& " p_{4}^{\mu}-\text { inlet" }: z_{I}=\infty_{I I} \longrightarrow f_{4}= \pm 1 \underset{\text { taken }}{=}-1
\end{aligned}
$$

By very good luck for obtaining the Veneziano model term hoped for these 4 inlets all lie on the same circle in the $f$-Riemann sphere, actually the unit circle since

$$
\left|f_{1}\right|=\left|f_{2}\right|=\left|f_{3}\right|=\left|f_{4}\right|=1 \text {. }
$$

This unit sphere can be considered (the boundary of) the Koba Nielsen disc.

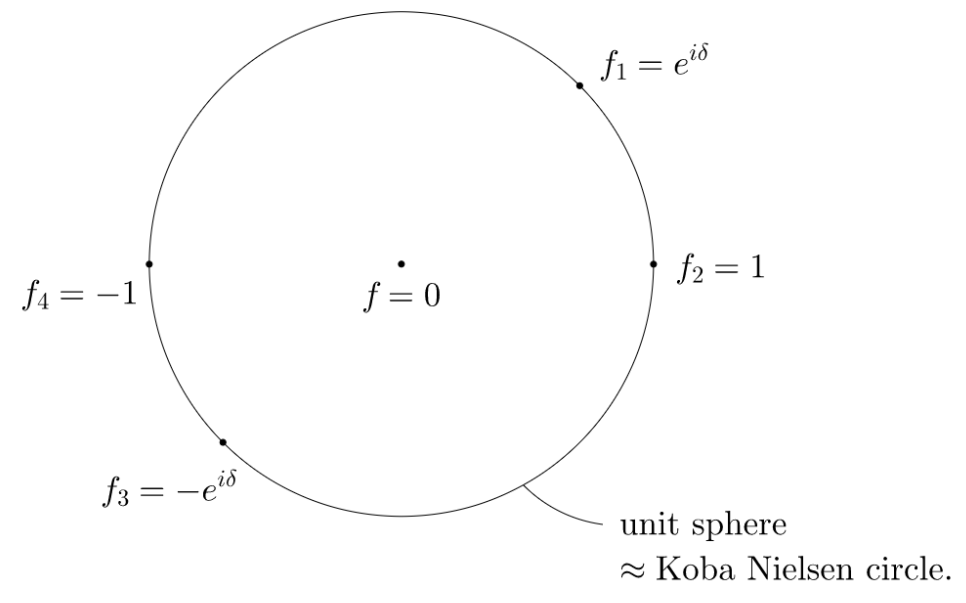

Figure 7.2 $f$-plane or $f$-Riemann surface.

In now calculating the heat production there is of course the little problem that taking the "inlets" as points there is a logarithmically divergent contribution from the neighborhood of the four inlet points. Since these inlet point divergencies are easily seen to give contribution in the exponent of the result for the functional integral proportional to the mass squares $m^{2}$ of the external particles they can be absorbed into the overall normalization of the amplitude, which we are not now sufficiently ambitions to compute anyway. One can of course in principle cut it off by making the inlets from small $\varepsilon_{i}$ radius circles around the ideal inlets.

One may also avoid seeing the divergences by not asking for the terms in the sum over the dimension proportional to the squares of the external $(25+1)$ momenta such as $p_{1}^{2}, p_{2}^{2}, p_{3}^{2}$ or $p_{4}^{2}$, but rather instead but instead for the terms proportional to inner products $p_{i}^{\mu} p_{j \mu}$ for $i \neq j$. For instance the usual Mandelstam variables contain these kinds of inner products as is well known

$$
\begin{aligned}
s & =\left(p_{1}+p_{2}\right)^{2}=\left(p_{3}+p_{4}\right)^{2}=m_{1}^{2}+m_{2}^{2}+2 p_{1} \cdot p_{2} \\
& =m_{3}^{2}+m_{4}^{2}+2 p_{3} \cdot p_{4}
\end{aligned}
$$




$$
t=\left(p_{1}-p_{4}\right)^{2}=\left(p_{2}-p_{3}\right)^{2}=m_{1}^{2}+m_{4}^{2}-2 p_{1} \cdot p_{4}=m_{2}^{2}+m_{4}^{2}-2 p_{2} \cdot p_{3}
$$

and

$$
u=\left(p_{1}-p_{3}\right)^{2}=\left(p_{2}-p_{4}\right)^{2}=m_{1}^{2}+m_{3}^{2}-2 p_{1} \cdot p_{3}=m_{2}^{2}+m_{4}^{2}-2 p_{1} \cdot p_{4} .
$$

In order to avoid having in mind all along the implication of $(25+1)$ momentum conservation

$$
p_{1}+p_{2}=p_{3}+p_{4}
$$

we might simply eliminate one of the external momenta, $p_{4}$ say,

$$
p_{4}=p_{1}+p_{2}-p_{3} .
$$

It is rather easy to calculate the heat production by means at computing of which electric potentials the currents are running in and out and using that all the energy going for the heat production must have been provided by the provision and take out of the current ( $\sim$ the momentum) at different electric potentials in the analogue model.

Because we at the end shall have $25+1$ momentum conservation we can without trouble start to evaluate the various potential contributions from the various $p_{i}$ 's as if the current from the inlet for string $i$ ran from this inlet for $i$ out to infinity in the f-plane (or rather f-Riemann surface) because at the end when all contributions are counted the net run out to $\infty$ will be zero because of momentum conservation.

To obtain the spectrum of the states match with the coefficient in the action for the functional integral we must in the analogue model as put forward by D. Fairlie and one of us [24] use the specific resistance $\pi \alpha^{\prime}$ (where $\alpha^{\prime}$ is the Regge-slope parameter). Now, however, we use in our cyclically ordered chain formalism of our novel string field theory a kind of "double" in the sense that e.g. an open string is covered by the cyclically ordered chain going both backward and forward. We can say that our cyclically ordered chains cover the strings doubly. When we now as in the present calculation use the cyclically ordered chain - which cover doubly - the specific resistance to be used should in stead of the old $\pi \alpha^{\prime}$ be $2 \pi \alpha^{\prime}$. In fact it must correspond to that a double layer of two conducting sheets, each with specific resistances $2 \pi \alpha^{\prime}$ will function like a single sheet with specific resistance $\pi \alpha^{\prime}$. This connection is of course nothing else than putting in parallel two resistances with resistance $2 \pi \alpha^{\prime}$ and getting a total resistance $\pi \alpha^{\prime}$.

The potential around an inlet say $f_{i}$ in the f-plane through which a current $p_{i}^{\mu}$ is pumped in/let in and leaves out at $f=\infty$ if we choose it to be zero for point $f$ in the distance 1 from $f$ is given as: Potential at the arbitrary point $f$ resulting from an inlet $p_{i}^{\mu}$ at $f_{i}$ is

$$
V_{\text {from } i}^{\mu}(f)=p_{i}^{\mu} \alpha^{\prime} \ln \left|f-f_{i}\right| \text {. }
$$

This is trivially calculated by noticing that the current at the distance $r=\left|f-f_{i}\right|$ from the inlet $f_{i}$ flows away from this $f_{i}$ with a current density $\frac{p_{i}^{\mu}}{2 \pi r}$ so that the potential variation with $\mathrm{r}$ must be

$$
\frac{\partial V_{\text {from } i}^{\mu}(f)}{\partial r}=2 \pi \alpha^{\prime} \cdot \frac{p_{i}^{\mu}}{2 \pi r}=\frac{\alpha^{\prime} p_{i}^{\mu}}{r}
$$


So the potential indeed integrates up

$$
V_{\text {from } i}^{\mu}(f)=\int_{1}^{r(f)=\left|f-f_{i}\right|} \frac{\alpha^{\prime} p_{i}^{\mu}}{r} d r=\alpha^{\prime} p_{i}^{\mu} \ln \left|f-f_{i}\right|
$$

and the total potential from all four inlets become in the notation that the outgoing strings 3 and 4 are written with extra minus on the current,

$$
\begin{aligned}
V^{\mu}(f)= & \alpha^{\prime}\left[p_{1}^{\mu} \ln \left|f-e^{i \delta}\right|+p_{2}^{\mu} \ln |f-1|\right. \\
& \left.-p_{3}^{\mu} \ln \left|f-\left(-e^{i \delta}\right)\right|-p_{4}^{\mu} \ln |f-(-1)|\right]
\end{aligned}
$$

Because of momentum conservation $p_{1}^{\mu}+p_{2}^{\mu}=p_{3}^{\mu}+p_{4}^{\mu}$ the amount of energy and there by the heat production is written in gauge invariant way as

$$
\text { -heat production rate }=\sum_{i=1,2} p_{i}^{\mu} V^{v}\left(f_{i}\right) g_{\mu v}-\sum_{i=3,4} p_{i}^{\mu} V^{v}\left(f_{i}\right) g_{\mu v}
$$

where of course

$$
g_{\mu \nu}=\left(\begin{array}{lllll}
1 & & & & \\
& -1 & & & \\
& & \ddots & & \\
& & & -1 & \\
& & & & -1
\end{array}\right) .
$$

Explicitly evaluated this becomes $4 \cdot 4=16$ terms of which 4 - namely the "diagonal ones" become divergent and could be expressed in terms of a cut off $\varepsilon$ as

$$
\sum_{i=1,2,3,4} \alpha^{\prime} p_{i}^{2} \ln \varepsilon
$$

The remaining 12 terms turn out to be pairwise identical and thus can be written as 6 terms

$$
\begin{aligned}
- \text { heat production rate }\left.\right|_{\text {off diagonal }}= & 2 \alpha^{\prime}\left[p_{1} \cdot p_{2} \ln \left|e^{i \delta}-1\right|\right. \\
& +p_{3} \cdot p_{4} \ln \left|-e^{i \delta}-(-1)\right| \\
& -p_{1} \cdot p_{4} \ln \left|e^{i \delta}-(-1)\right| \\
& -p_{2} \cdot p_{3} \ln \left|1-\left(-e^{i \delta}\right)\right| \\
& -p_{1} \cdot p_{3} \ln \left|e^{i \delta}-\left(-e^{i \delta}\right)\right| \\
& \left.-p_{2} \cdot p_{4} \ln |1-(-1)|\right]
\end{aligned}
$$

As we have assumed the masses of the external strings all to be same, namely the tachyonic mass with square

$$
m_{i}^{2}=-\frac{1}{\alpha^{\prime}}(\text { for } i=1,2,3,4)
$$


we have indeed (using also momentum conservation)

$$
\begin{aligned}
& p_{1} \cdot p_{2}=p_{3} \cdot p_{4} \\
& p_{1} \cdot p_{4}=p_{2} \cdot p_{3}
\end{aligned}
$$

and

$$
p_{1} \cdot p_{3}=p_{2} \cdot p_{4}
$$

Using this we reduce further the expression for the finite or "off diagonal" part of minus the heat production

$$
\begin{aligned}
& \text {-heat production rate }\left.\right|_{\text {off diagonal }}=4 \alpha^{\prime} p_{1} \cdot p_{2} \ln \left|e^{i \delta}-1\right|-4 \alpha^{\prime} p_{1} \cdot p_{4} \ln \left|e^{i \delta}+1\right| \\
& -4 \alpha^{\prime} p_{1} \cdot p_{3} \ln 2 \\
& =4 \alpha^{\prime} p_{1} \cdot p_{2} \ln \left|\sin \frac{\delta}{2}\right|-4 \alpha^{\prime} p_{1} \cdot p_{4} \ln \left|\cos \frac{\delta}{2}\right| \\
& +4 \alpha^{\prime}\left(p_{1} \cdot p_{2}-p_{1} \cdot p_{4}-p_{1} \cdot p_{3}\right) \ln 2
\end{aligned}
$$

Noticing that

$$
p_{1} \cdot p_{2}-p_{1} \cdot p_{4}-p_{1} \cdot p_{3}=-p_{1}^{2}=-m_{1}^{2}=\frac{1}{\alpha^{\prime}}
$$

the last term in (7.29) after is seen to be just an insignificant constant $4 \ln 2$.

Since the $\sin \frac{\delta}{2}$ and $\cos \frac{\delta}{2}$ are actually written with a $\delta$ chosen positive it would have be more correct to have written then as $\left|\sin \frac{\delta}{2}\right|$ and $\left|\cos \frac{\delta}{2}\right|$ even; and since squring them is for the logarithm is only a factor 2 we shall prefer to write the non-constant terms

$$
\begin{array}{r}
- \text { heat production rate }\left.\right|_{\text {non-constant }}=- \text { heat production rate }\left.\right|_{\text {off diagonal }}-4 \ln 2 \\
=2 \alpha^{\prime}\left[p_{1} \cdot p_{2} \ln \left[\left(\sin \frac{\delta}{2}\right)^{2}\right]-p_{1} \cdot p_{4} \ln \left[\left(\cos \frac{\delta}{2}\right)^{2}\right]\right]
\end{array}
$$

Here we notice that the arguments for logarithms have turned out - rather likely for the Veneziano model - to obey the rule

$$
\sin ^{2} \frac{\delta}{2}+\cos ^{2} \frac{\delta}{2}=1
$$

so that if we introduce a new variable

$$
X=\sin ^{2} \frac{\delta}{2}
$$

we have

$$
\cos ^{2} \frac{\delta}{2}=1-X
$$


Also note that from our geometrical representation and the way must integrate over $\delta$ namely in the range

$$
0 \leq \delta \leq \pi
$$

the new variable $X=\sin ^{2} \frac{\delta}{2}$ comes to run through the range

$$
0 \leq X \leq 1 .
$$

The measure in the $\mathrm{X}$-notation is related to the one in $\delta$-notation by

$$
d X=d \sin ^{2} \frac{\delta}{2}=\sin \frac{\delta}{2} \cos \frac{\delta}{2} d \delta
$$

so that the from the counting of objects as the measure to be used measured $\delta$ becomes

$$
d \delta=\frac{d X}{\sin \frac{\delta}{2} \cos \frac{\delta}{2}}=\frac{d X}{\sqrt{X(1-X)}} .
$$

Thus we obtain apart from an overall multiplicative constant that the amplitude contribution in which 1 and 2 have annihilating parts as well as 3 and 4 and pieces go from 1 to 4 and from 2 to 3 , while no direct transfer between 1 and 3 nor 2 and 4 the expression

$$
\begin{aligned}
\text { Ampl }_{\text {s.t channel }} & =\left.\int_{0}^{1} e^{- \text {heat prod rate }}\right|_{\text {non constant }} \frac{d X}{\sqrt{X(1-X)}} \\
& =\int_{0}^{1} X^{2 \alpha^{\prime} p_{1} \cdot p_{2}}(1-X)^{-2 \alpha^{\prime} p_{1} \cdot p_{4}} \frac{d X}{\sqrt{X(1-X)}}
\end{aligned}
$$

To make this expression look a bit more familiar we can of course use

$$
\begin{aligned}
& \alpha^{\prime} 2 p_{1} \cdot p_{2}=\alpha(s)-\alpha^{\prime} \cdot\left(m_{1}^{2}+m_{2}^{2}\right)-\alpha(0)=\alpha(s)+1 ; \\
& -2 \alpha^{\prime} p_{1} \cdot p_{4}=\alpha(t)-\alpha^{\prime} \cdot\left(m_{1}^{2}+m_{2}^{2}\right)-\alpha(0)=\alpha(t)+1 ;
\end{aligned}
$$

and write the amplitude contribution for the $(\mathrm{t}, \mathrm{s})$-channels so to speak

$$
A m p l_{\text {s.t channel }}=\int_{0}^{1} X^{\alpha(s)+1}(1-X)^{\alpha(t)+1} \frac{d X}{\sqrt{X(1-X)}}
$$

It should be had in mind that we did here simply ignore the anomaly.

In our earlier articles [18] [19] we used a notation of calling $\delta$ what was here called $2 \delta$ and calculated that the effect of the anomaly were to change the functional integral by a correction factor the logarithm of which was (see $(81,82)$ in that article)

$$
\begin{aligned}
\Delta & \text { anomaly } \ln \text { integrand } \\
= & \Delta_{\text {anomaly }}(- \text { heat production rate }) \\
= & \text { " } \delta-\text { independent part" } \\
& \quad+(d-2) \frac{1}{96 \pi} \cdot \frac{1}{2} \ln \sin 2 \delta \cdot(4 \pi+4 \pi) \\
= & \frac{d-2}{24} \ln \sin 2 \delta(\bmod \text { constants }) \\
= & \frac{d-2}{24} \ln \sqrt{X(1-X)}(\text { mod constants })
\end{aligned}
$$


where $\mathrm{d}$ is the dimension of space-time and $d-2$ the number of say "transverse dimensions" (or better the number of dimensions ones the degrees of freedom of as many as can be gauge fixed have been gauge-fixed). This correction gives then to the integrand or the functional integral a correction factor

$$
\begin{aligned}
& \exp \left(\Delta_{\text {anomaly }}(- \text { heat production rate })\right) \\
& =\exp \left(\frac{d-2}{24} \ln \sqrt{X(1-X)}\right) \\
& =[X(1-X)]^{\frac{d-2}{48}} .
\end{aligned}
$$

Thus our for anomaly uncorrected expression

$$
\text { Ampl }_{s, t c h a n n e l}=\int_{0}^{1} X^{-\alpha(s)-1}(1-X)^{-\alpha(t)-1} \frac{d X}{\sqrt{X(1-X)}}
$$

get

$$
=\int_{0}^{1} X^{-\alpha(s)-1}(1-X)^{-\alpha(t)-1}(X(1-X))^{\frac{d-2}{48}-\frac{1}{2}} d X
$$

In the usual form of the Veneziano amplitude term $A m p l_{\text {s.tchannels }}$

$$
\begin{aligned}
\left.A m p l_{\text {s.t channels }}\right|_{\text {usual }} & =B(-\alpha(s),-\alpha(t)) \\
& =\int_{0}^{1} X^{-\alpha(s)-1}(1-X)^{-\alpha(t)-1} d X
\end{aligned}
$$

the factor of the form

$$
(X(1-X))^{\frac{d-2}{48}-\frac{1}{2}}
$$

is missing. Thus to reproduce this simple "usual" form the power to the product $X(1-X)$ namely

$$
\frac{d-2}{48}-\frac{1}{2}
$$

should be made 0 . It is of course obvious that this power becomes just 0 for the anomaly cancellation required dimension $d=26$. It should really be taken as a special suggestion that our novel string field theory really is a string theory. That it just goes correct just for $d=26$ dimensions.

But for $d=26$ dimensions - meaning really $25+1$ - we have now gotten out of our novel string field theory, i.e. our object formulation plus appropriate wave functions for the strings expressed in terms of objects, the correct term for $A_{m p l}$ s.t channels (apart though from the overall factor). In the earlier paper [18] [19] we already found the term corresponding having resonances in the $\mathrm{u}$ - and the $\mathrm{t}$ - channels. The term with resonances in the channels(s, u) is completely analogous to the (s, t) - term derived above.

So indeed we got all the three terms expected. So now we really proved that for the four particle amplitude apart from the overall constant factor we really got the full Veneziano amplitude 
out of our novel string field theory. Overlap between initial and final string states in the object formulation gives the usual string theory!

This fact should strongly encourage the belief in that our novel string field theory - i.e. the "free" object model - is indeed a reformulation of string theory.

\section{Conclusion and outlook}

We develop a new description for an arbitrary number of strings, a string field theory.

It is formulated in terms of a discretization into pieces - much like Thorn's string bits, but we do it for right and left movings- components and the then string bits of thorn are then called by us "objects".

These objects have dynmmics like free massless particles. That is to say they are decided by a quantum field theory of free massless particle. In momentum space they are static.

So nothing happens, even if the strings scatter!

We have arguments that our model is really a transformation of theory for several strings.

As for deriving the Veneziano model, first with some troubles, but having negative even energy for the objects will presumably help to get the full Veneziano model (we missed two terms at first)

Also the spectrum we got o.k., except for a species doubler problem[21], [22].

(Apart from null sets) our string field theory should be just a rewriting of usual say string field theory.

The Hilbert space describing all the possible states in a string world is the Fock space of -either one or two- theories of massless noninteracting scalars(for the bosonic $25+1$ model).

Two massless free scalar theories/species of scalar particles for purely closed string theory, while only one when there are open strings.

But allowed states are restricted to obey -approximately- some "chiral" invariant continuity condition: this means that the stringyness only comes in via initial state conditions.

We think we have a new(novel) way of representing string theory, which because of being in some respects simpler could be helpful in understanding some aspects of string theory better.

Even if string theory should not turn out to be the final truth -as can still be the case- its abilities for providing a cut off are so good that alone in looking for cut off it may give inspiration.

It happens generally thinking to seek a cut off you easily get in the direction of the string theory, especially the aspect of not having any true interaction as is a trademark for OUR NOVEL SFT MODEL.

Our novel field theory deviates from usual ones - Kaku Kikkawa's or Witten's by including (a nul set of) of information less in its description of state of the world, i.e. of a set of strings present. We have rewritten the information - the kept part - on a state of several strings into a state of something (more like particles), which we call "objects", to such a degree that one only sees the connection to genuine strings by quite a bit of complicated rewriting. 
Our novel string field theory is genuinely nonperturbative theory. We should be able to redenve nonperturbative theory of string theories such as branes. Also so fan the background space time is flat. Next step will be taking non flat, e.q. pp-wave background.

Now if our string field theory (and string theory) is the theory of everything (TOE), we should be able to derive inflation theories in early universe: it may be one of the greatest challenge which we are planning to attack.

In very high energies such as Planck scale and/or string scale, we may be able to investigate truly new physics, for instance, studying supersymmetric particles.

\section{Acknowledgements}

The authors thank K. Murakami, K. Sugiyama, M. Sakaguchi and Y. Sekino for their useful comments.

One of us (H. B. N.) acknowledges the Niels Bohr Institute for allowance to work as emeritus. M. Ninomiya acknowledges Osaka city University, Advanced Mathematical Institute supporting this work. the Niels Bohr Institute and the Niels Bohr International Academy for giving him very good hospitality during his stay. M.N. also acknowldges the present research is supported in part by the JSPS Grant in Aid for Scientific Research No. 15K05063.

H.B.N. thanks to the Bled Conference participants, organizers and Matiaz Breskov for finantial support to come there where many of the ideas of this work got tested.

\section{A. What is the Rough Dirac Sea?}

In a free theory of second quantized fermions it is well known that the negative energy single fermion states are all filled while the positive energy single fermion states are all empty.

When there are interactions between such fermions or with other fields the ground state is no longer so simple. The vacuum is in this case rather a superposition of a lot of free energy states of the second quantized theory, a lot of which have empty single fermion negative energy states, or filled positive single fermion states.

This is analogous to that in a peaceful sea there is water for negative height and air for eigenstate positive height.

In a rough sea there is near height zero almost equal probability for finding water and air.

So if you act with an annihilation operator for a positive energy single fermion state or with a negative single fermion creation operator on a vacuum with interaction, then you obtain a state, in which the sum of the single fermion energies (ignoring the interaction) has been lowered.

The interacting vacuum is by definition the lowest energy state, when the interaction is included, but it is not the lowest energy state for the free fermion energy, so the free fermion energy can easily be lowered by some annihilation of a positive energy fermion or creation of a negative energy one. This is analogous to that you could remove a droplet of water from a positive height position from a rough sea; or you could add a droplet in a negative height place, with some slight amount of luck only needed. 


\section{A.1 The Idea of the Rough Dirac Sea}

Really what we have in mind in the case of usual (particle) quantum field theory under the notation of the "Rough Dirac Sea" is just the true vacuum of the quantum field theory in the case of a strongly interacting theory. In a free quantum field theory one has a Dirac sea in which just all states with negative energy are filled while those with positive energy are empty so that

$$
\begin{aligned}
a(\vec{p}, E>0)|0\rangle & =0 \\
a^{+}(\vec{p}, E>0)|0\rangle & \neq 0
\end{aligned}
$$

and

$$
\begin{aligned}
& a_{\text {anti }}(\vec{p}, E>0)|0\rangle=a^{+}(-\vec{p},-E<0)|0\rangle=0 \\
& a_{\text {anti }}^{+}(\vec{p}, E>0)|0\rangle=a(-\vec{p},-E<0)|0\rangle \neq 0 .
\end{aligned}
$$

But now if there are interactions the true vacuum get much more complicated and one could obtain it by a development of some state, e.g. the bare vacuum $|0\rangle$ through a long imaginary time so that the propagation operator becomes

$$
e^{-H_{\text {large }}}
$$

where $t_{\text {large }}$ is a very large time. Then we get the true vacuum

$$
|0\rangle_{\text {true }} \propto \lim _{t_{\text {large }}} \rightarrow \infty e^{-H_{t_{\text {large }}}}|0\rangle .
$$

If the interactions in Hamiltonian $H$ are strong the true vacuum is very much different from the bare one $|0\rangle$ Then it will be so that all operations with bare creation and annihilation operators $a^{+}(\vec{p}, E \gtrless 0)$ and $a(\vec{p}, E \gtrless 0)$ will give different from zero results when acting on $|0\rangle_{\text {true }}$.

I.e.

$$
\begin{array}{r}
a(\vec{p}, E>0)|0\rangle_{\text {true }} \neq 0 \\
a^{+}(\vec{p}, E>0)|0\rangle_{\text {true }} \neq 0 \\
a(\vec{p}, E<0)|0\rangle_{\text {true }} \neq 0 \\
a^{+}(\vec{p}, E<0)|0\rangle_{\text {true }} \neq 0 .
\end{array}
$$

Thus it is quite easy seemingly to add to the true vacuum negative energy. This is, however, only true when one thinks of the free approximation energy $H_{0}$, then one has

$$
\left\langle 0_{\text {true }}\left|a(\vec{p}, E<0) H_{0} a^{+}(\vec{p}, E<0)\right| 0_{\text {true }}\right\rangle<\left\langle\left. 0\right|_{\text {true }} H_{0} \mid 0\right\rangle_{\text {true }}
$$

But using true Hamiltonian $H$ instead of $H_{0}$ would mean that the true vacuum is the lowest eigenstate so that

$$
\langle b|H| b\rangle \geq\left\langle\left. 0\right|_{\text {ture }} H \mid 0\right\rangle_{\text {true }}
$$

for any state $|b\rangle$, also for

$$
|b\rangle=a^{+}(\vec{p}, E<0)|0\rangle_{\text {true }} .
$$


Even if we decide to make the here considered fermion be a Majorana fermion so that on the allowed states, the allowed subspace of the (second quantized) Hilbert space we have

$$
a_{\text {anti }}^{+}(\vec{p}, E>0) \text { effectively } a^{+}(\vec{p}, E>0) .
$$

We can still have this seeming - i.e. w.r.t. $H_{0}$ - addition of negative energy.

In our present work we want to use the story of the "Rough Dirac sea" for the objects $(\sim$ bits of strings taken in the $X_{R}$ and $X_{L}$ instead of Thorns fullX) - which in our scheme are essentially particles.

In our model we have, however, no interaction between objects and so a genuine true vacuum is not obvious to define. We so to speak lack $H$ just above. However, we can without really explaining it assume that there exist some especially selected - by God - background state which we can identify with the true vacuum for the objects $|0\rangle_{\text {true }}($ for $o b j$ ) and then we should think of replacing the at first presented $\mid 0>$ as vacuum for the objects by the more complicated $|0\rangle_{\text {true }}($ for obj $)$

$$
|0\rangle \longrightarrow|0\rangle \text { true }(\text { for obj })
$$

Acting on this $|0\rangle_{\text {true (for } o b j)}$ we can now seemingly add both negative and positive energy. In this way one can on $|0\rangle_{\text {true }}($ for $o b j$ ) as background create states which have negative energy density along the cyclic chains, and they can be removed again by a positive energy preation operator series: e.g.

$$
\prod_{I} a^{+}\left(J^{\mu}(I)\right)|0\rangle \text { true (for obj) }
$$

has compared $|0\rangle_{\text {true }}$ (for obj) itself a negative "bare" energy $\Sigma_{J^{0}}$ if the $J^{\mu}$ 's obey

$$
J^{0}(I)<0
$$

\section{B. Use of Rough Dirac sea Analogy for Our Novel String Field Theory Background State}

Although we do not have any genuine interaction between the objects in our model, we shall nevertheless imagine that the "background state" on which we act with object-creation and objectannihilation operators is a complicated state, so that it is not the ground state for the sum of the single object states, so that it is not a problem to act with some annihilation or creation operator so as to add negative free energy, meaning make the sum of the object energies more negative.

\section{References}

[1] String references are cited in References [12]-[15], [16]

H. B. Nielsen, A Physical interpretation of the integrand of the n-point Veneziano model, (1969)(Nordita preprint...); a later version "An almost Physical interpretation of the integrand of the $n$-point Veneziano model" preprint at Niels Bohr Institute; a paper presented at the 15th International Conference on High Energy Physics, Kiev 1970 (see p.445 in Veneziano's talk.) 
[2] Y. Nambu, Quark Model and the factorization of the Veneziano amplitude, in Proceedings of the International Conference on Symmetries and Quark Models, June 18-20, 1969 ed. by Chand, R. (Gordon and Breach), New York, 269-277; reprinted in Broken Symmetry Selected Papers of Y. Nambu eds. T. Eguchi and K. Nishijima, (World Scientific, Singapore, 1995)258-277.

[3] Y. Nambu, Duality and hadrondynamics, Lecture notes prepared for Copenhagen summer school, 1970; It is reproduced in "Broken Symmetry", Selected Papers of Y. Nambu eds. T. Eguchi, and K. Nishijima, (World Scientific, Singapore, 1995)280.

[4] L. Susskind, Structure of hadrons implied by duality, Phys. Rev. D1(1970)1182-1186(1970): L. Susskind, Dual symmetric theory of hadrons, 1. Nuovo Cimento A69,457-496(1970).

[5] For many original papers of early string theory found in The Birth of String Theory" eds. by Andrea Cappeli, Elena Castellani, Flippo Colomo and Paolo Di Vecchia, Cambridge University Press 2012. Cambridge, New York, Melbourne.

[6] M. B. Green, J. H. Schwarz and E. Witten, Superstring theory, 1-2, Cambridge University Press 1987.

[7] For comprehensive reviews of string theory see e.g. J. Polchinski, String Theory, vol.I-II, Cambridge University Press 1998.

[8] For review articles of string field theory:

W. Taylor and B. Zwiebach, D-branes, Tachyons, and String Field Theory, hep-th/0311017 http://jp.arxiv.org/abs/hep-th/0311017; K. Ohmori, A Review on Tachyon Condensation in Open String Theories, hep-th/0102085 http://jp.arxiv.org/abs/hep-th/0102085.

[9] As for bosonic string field theory in the light-cone gauge:

M. Kaku and K. Kikkawa, Phys. Rev. D10(1974)1110; M. Kaku and K. Kikkawa, Phys. Rev. D10(1974)1823; S. Mandelstam, Nucl. Phys. B64(1973)205; E. Cremmer and J.-L. Gervais, Nucl. Phys. B90(1975)410.

[10] Joining-Splitting light-cone type: for open string H. Hata, K. Itoh, T. Kugo, H. Kunitomo and K. Ogawa, Phys. Rev. D34(1986)2360.

[11] Closed string of Joining-Splitting light-cone type:

H. Hata, K. Itoh, H. Kunitomo and K. Ogawa, Phys. Rev. D35(1987)1318;

H. Hata, K. Itoh, T. Kugo, H. Kunitomo and K. Ogawa, Phys. Rev. D35(1987)1356.

[12] Supersymmetrized Green-Schwarz theory in the light-cone gauge:

M. B. Green and J. H. Schwarz, Nucl. Phys. B218(1983)43; M. B. Green, J. H. Schwarz and L. Brink, Nucl. Phys. B219(1983)437; M. B. Green, and J. H. Schwarz, Nucl. Phys. B243(1984)437; J. Greensite and F. R. Klinkhamer, Nucl. Phys. B281(1987)269; Nucl. Phys. B291(1987)557; Nucl. Phys. B304(1988)108; M. B. Green and N. Seiberg, Nucl. Phys. B299(1988)559; S.-J. Sin, Nucl. Phys. B313(1989)165.

[13] Witten type mid-point interaction of covariant bosonic string field theory: for open string E. Witten, Nucl. Phys. B268(1986)253.

[14] Closed string version of ref.[4] of the Witten theory:

M. Saadi and B. Zwiebach, Ann. Phys. 192(1989)213; T. Kugo, H. Kunitomo and K. Suehiro, Phys. Lett. B226(1989)48; T. Kugo and K. Suehiro, Nucl. Phys. B337(1990)434; B. Zwiebach, Nucl. Phys. B390(1993)33.

[15] Supersymmetrized covariant open Witten theory

E. Witten, Nucl. Phys. B276(1986)291; I. Arefeva and P. Medvedev, Phys. Lett. B212(1988)299;

C. Wendt, Nucl. Phys.B314(1989)209; N. Berkovits, Nucl. Phys. B450(1995)90 [Errata, B459(1996)439] hepth/9503099. 
[16] Modified cubic theory:

I. Arefeva, P. Medvedev and A. Zubarev, Nucl. Phys. B341(1990)464; C. R. Preitschopf, C. B. Thorn and S. A. Yost, Nucl. Phys. B337(1990)363.

[17] H. B. Nielsen and M. Ninomiya, A New Type of String Field Theory, in Proceedings of the 10th Tohwa International Symposium in String Theory", July 3-7, 2001, Fukuoka Japan, AIP conference Proc vol.607, p.185-201; arXiv: hep-th/0111240.v1, Nov.2001

[18] H. B. Nielsen and M. Ninomiya, An Idea of New String Field Theory - Liberating Right and Left movers -, in Proceedings of the 14th Workshop, "What Comes Beyond the Standard Models" Bled, July 11-21, 2011, eds. N. M. Borstnik, H. B. Nielsen and D. Luckman; arXiv: 1112. 542[hep-th].

[19] H.B. Nielsen and M. Ninomiya, “A Novel String Field Theory Solving String Theory by Liberating Left and Right Movers” JHEP_202P_05131 v2 (2013)

[20] C. B. Thorn, Derivational model from field theory II, Phys. Rev. D17(1978)1037, and references therein.

[21] G. Veneziano, Construction of a crossing-symmetric, Regge behaved amplitude for linearly rising trajectories, Nuovo Cimento A57 190(1968).

[22] Appearance of doubling, K. G. Wilson, Confinement of quarks, Phys. Rev. D10 2445(1974), see also H. B. Nielsen and M. Ninomiya, Absence of neutrinos on a lattice I - Proof by homotopy theory-.

[23] Z. Koba and H. B. Nielsen, Nucl. Phys. B12 243(1969).

[24] D. B. Fairlie and H. B. Nielsen Nucl. phys B20 (1970) 637-651 